\title{
Mecanismos de Desconexión Moral, Diferencias de Sexo y Predictores Clínicos en Adolescentes: Un Estudio Exploratorio
}

\section{Moral Disengagement Mechanisms, Sex Differences, and Clinical Predictors in Adolescents: An Exploratory Study}

\author{
Anyerson Stiths Gómez-Tabares ${ }^{1}$, César Núñez ${ }^{2}$ y Vicente E. Caballo ${ }^{3}$ \\ ${ }^{1}$ Facultad de Psicología y Ciencias Sociales, Universidad Católica Luis Amigó \\ 2 Facultad de Ciencias Sociales y Humanas, Universidad de Medellín \\ ${ }^{3}$ Facultad de Psicología, Universidad de Granada
}

\begin{abstract}
La desconexión moral es un constructo útil para explicar el comportamiento disruptivo en la adolescencia, evidenciando diferencias entre hombres y mujeres. Sin embargo, no existen estudios respecto de la asociación de factores clínicos y psicológicos y el uso de mecanismos de desconexión moral. Por tal motivo, este estudio analizó las diferencias de sexo y predictores clínicos de los mecanismos de desconexión moral en adolescentes. Se utilizó una metodología cuantitativa, no experimental, transversal y explicativa, con la participación de 354 adolescentes entre 14 y 18 años $(M=15,58, D E=1,22)$ de dos instituciones educativas de Manizales, Colombia. Se utilizó el cuestionario de desconexión moral (MMDS) y las escalas de autoestima (RSES), impulsividad (BIS-11), desesperanza (BHS), ansiedad (BAI) y depresión (BDI). Una prueba $t$ de Student mostró que los hombres presentan puntuaciones más altas que las mujeres en todos los mecanismos de desconexión moral. Un análisis de regresión lineal múltiple por sexo también reveló que la impulsividad tuvo un efecto sobre la desconexión moral en hombres, mientras que las variables impulsividad, desesperanza y ansiedad evidenciaron los efectos más altos que explican la desconexión moral en mujeres. El modelo de regresión lineal múltiple evidenció que la impulsividad y el sexo presentaron los efectos más altos en la predicción de la desconexión moral y sus mecanismos. Se considera que los resultados aportan explicaciones teóricas novedosas en una línea de investigación que pretende relacionar las perspectivas psicológicas, clínicas y cognitivo-sociales con implicaciones importantes para la intervención psicosocial en adolescentes.
\end{abstract}

Palabras clave: desconexión moral, depresión, ansiedad, impulsividad, adolescente

Moral disengagement is a useful construct for explaining disruptive behavior in adolescents, revealing differences between men and women. However, no research has been conducted on the association between clinical and psychological factors and the use of moral disengagement mechanisms. For this reason, this study analyzed sex differences and clinical predictors of moral disengagement mechanisms in teenagers. A quantitative, nonexperimental, cross-sectional, and explanatory methodology was used. The participants were 354 adolescents between 14 and 18 years of age $(M=15.58, S D=1.22)$ from two educational institutions in Manizales, Colombia. The moral disengagement scale (MMDS) was used along with self-esteem (RSES), impulsivity (BIS-11), despair (BHS), anxiety (BAI), and depression scales (BDI). A Student's t-test showed that men scored higher than women on all moral disengagement mechanisms. A multiple linear regression analysis by sex also revealed that impulsivity had an effect on moral disengagement in men, whereas the variables impulsivity, despair, and anxiety showed the highest effects explaining moral disengagement in women. The multiple linear regression model showed that impulsivity and sex had the strongest effects on the prediction of moral disengagement and its mechanisms. The results are considered to offer innovative theoretical explanations in a line of research aimed at linking psychological, clinical, and cognitive-social perspectives, with major implications for psychosocial intervention in adolescents.

Keywords: moral disengagement, depression, anxiety, impulsivity, adolescent

Anyerson Stiths Gómez-Tabares (D) https://orcid.org/0000-0001-7389-3178

César Núñez (D) https://orcid.org/0000-0001-8925-993X

Vicente E. Caballo (D) https://orcid.org/0000-0002-2767-8028

Este estudio recibió apoyo financiero de la Universidad Católica Luis Amigó, Colombia, y se desarrolló en colaboración con la Universidad de Medellín y la Universidad de Granada. No existe ningún conflicto de intereses que revelar.

La correspondencia relativa a este artículo debe ser dirigida a Anyerson Stiths Gómez Tabares, Programa de Psicología, Facultad de Psicología y Ciencias Sociales, Universidad Católica Luis Amigó, Carrera $22 \mathrm{~N}^{\circ}$ 67A - 49, Manizales, Colombia. Email: anyerspn.gomezta@amigo.edu.co 
La teoría de la desconexión moral propuesta por Bandura (1990, 1991, 1999, 2002) se ha considerado en las últimas décadas un marco de referencia para explicar la conducta social desadaptativa, especialmente en la niñez y la adolescencia. La conducta social está influenciada por procesos de autorregulación sociocognitiva que les permite a las personas actuar de manera correcta o incorrecta en función de los valores y principios morales a nivel individual y social (Gómez Tabares, Narváez Marín et al., 2019). De manera particular, se ha encontrado que la conducta antisocial y violenta se ha asociado a la desvinculación de estos procesos cognitivo-regulatorios a nivel ético-moral (Bandura et al., 1975; Hardy et al., 2015; Hyde et al., 2010).

La desconexión moral es una estrategia de reconstrucción sociocognitiva con la cual una persona justifica una acción inmoral para evitar la autocensura y la disonancia cognitiva cuando actúa contrariamente a los criterios ético-morales; constituye así un proceso de reformulación de la conducta trasgresora como algo moralmente aceptable (Bandura, 1999; Doyle \& Bussey, 2018; Hymel \& Perren, 2015). En este sentido, las personas generalmente son conscientes de que sus acciones violentas o antisociales están a contramano de los estándares morales a nivel social, y hacen uso de la desconexión moral como una estrategia para alterar la comprensión de sus acciones, justificarlas y hacerlas ver como aceptables y no censurables (D'Urso et al., 2018).

$\mathrm{Al}$ respecto, los procesos cognitivos de autorregulación asociados a la autocensura y la culpa se pueden eludir mediante el uso de uno o varios de estos mecanismos de desconexión (Bandura, 2002; Gini et al., 2011; Petruccelli et al., 2017). Para Gutzwiller-Helfenfinger (2015), los estándares morales regulatorios asociados a la autovaloración negativa de la conducta violenta se desconectan selectivamente y, de este modo, se legitima la acción inmoral como justificable, evitando la disonancia cognitiva entre el sentido y los estándares morales a nivel social.

La desconexión moral se compone de ocho mecanismos cognitivos, segmentados en tres grandes dominios psicológicos (Bandura, 1999, 2002, 2016; Rubio-Garay et al., 2017). Al primer dominio se le denomina conducta reprobable, la cual comprende los mecanismos de justificación moral, lenguaje eufemístico y comparación ventajosa. Este dominio hace referencia a la reconstrucción de la percepción valorativa de la conducta perjudicial, con el fin de que no se considere inmoral. El segundo dominio son los efectos negativos o perjudiciales, consistentes en tergiversar las consecuencias de la conducta para que parezca menos nociva, a la vez que busca minimizar la agencia de acción de la persona por el daño causado. En este domino se encuentran los mecanismos de distorsión de las consecuencias y, en la intersección entre el primer y segundo dominio, se integran el desplazamiento y difusión de la responsabilidad. El último dominio, denominado víctima, consiste en devaluar a las víctimas, despojándolas de su calidad como seres humanos o culpándolas por el daño recibido, y para hacerlo se utilizan los mecanismos de deshumanización y atribución de la culpa. De estos dominios se desprenden los ocho mecanismos de desconexión moral mencionados, que se explican a continuación:

Justificación moral: las personas generalmente no son capaces de ejecutar conductas dañinas, porque son conscientes de las consecuencias o autosanciones que estas representan, por lo cual requieren de justificaciones morales para convencerse a sí mismos y sentirse libres de la autocensura, con el fin de que esta conducta logre ser aprobada moral y socialmente.

Lenguaje eufemístico: el lenguaje es la estructura por la cual las personas dan vida a sus pensamientos y la manera cómo actúan. Dichas acciones pueden ser vistas de una forma agradable o repugnante, dependiendo de cómo se le llamen. Es aquí donde el lenguaje o etiquetado eufemístico entra en juego para ocultar lo cruel de una conducta dañina y hacerla más respetable ante los ojos de los demás, dándole un nombre y un sentido más benigno y socialmente aceptado.

Comparación ventajosa: en este mecanismo se encuentra el principio de contraste, en el cual una conducta dañina puede hacerse más benevolente, dependiendo del hecho o comportamiento con el cual sea contrastado de manera conveniente, es decir, entre más trágica e inhumana sea la conducta comparada, más aceptable, incluso benevolente, será la propia conducta inmoral (Bandura, 1990, 1999, 2002; Gómez Tabares \& Narváez Marín, 2019; Kollerová et al., 2018).

Desplazamiento de la responsabilidad: hace parte del segundo conjunto de mecanismos de desconexión moral. Consiste en minimizar la culpa generada por la conducta dañina, dirigiendo la responsabilidad a los demás. Aquí, las personas hacen que las consecuencias de sus actos sean producto de las órdenes de otros, como lo puede ser un superior, exonerándose de cualquier sentimiento de culpa o responsabilidad moral. 
Difusión de la responsabilidad: se atribuye la responsabilidad de las propias acciones reprensibles al grupo, como consecuencia del comportamiento colectivo. Mediante este mecanismo las personas pueden actuar de manera cruel, atribuir dicho comportamiento a las decisiones grupales y evitar la responsabilidad personal (Bandura, 1990, 1999, 2002; Gómez Tabares, Núñez et al., 2019; Kollerová et al., 2018).

Distorsión de las consecuencias: "los daños ocasionados por una conducta se ignoran, malinterpretan o minimizan, evitando que se active la autocensura moral" (Gómez Tabares \& Narváez Marín, 2019, p. 606). Si esto no es suficiente, se pasa a desacreditar la consecuencia para obtener un beneficio personal.

Deshumanización: consiste en considerar a la víctima como carente de cualidades humanas, cruel o salvaje, con el fin de justificar el daño hacia ella. Bajo este mecanismo, se legitiman conductas violentas e inhumanas hacia otras personas, incluyendo acciones bélicas y de lesa humanidad (Bandura, 1999, 2002; Gómez Tabares \& Narváez Marín, 2019; Kollerová et al., 2018; Villegas de Posada et al., 2018).

Atribución de la culpa: el sujeto se victimiza a tal punto de convencerse de que su conducta dañina fue provocada por los demás. La persona se representa como una víctima obligaba a ejercer una conducta perjudicial hacia alguien, resultado de una provocación forzosa, atribuyendo la culpa a la verdadera víctima (Bandura, 1990, 1999, 2002; Kollerová et al., 2018).

La desconexión moral y sus respectivos mecanismos han servido para explicar la conducta violenta y antisocial en la infancia y la adolescencia. Diversos estudios han encontrado de manera consistente una relación entre el uso de la desconexión moral y el comportamiento agresivo, violento y antisocial, el acoso escolar, la actividad delictiva y la intimidación física y verbal en adolescentes (Bussey et al., 2015; Gini et al., 2014; Gini et al., 2015; Hardy et al., 2015). Al respecto, el metaanálisis realizado por Gini et al. (2014) muestra que la desconexión moral es un predictor de la conducta agresiva y antisocial. A mayor desconexión moral las expresiones de agresión y comportamiento antisocial se incrementan, a la vez que disminuye la disposición a experimentar empatía por sus víctimas (D'Urso et al., 2018; Gómez Tabares \& Narváez Marín, 2019; Kokkinos \& Kipritsi, 2018).

También se ha encontrado de manera consistente que la desconexión moral es un inhibidor de la empatía, la prosocialidad y la sensibilidad por el bienestar de los demás (Bussey et al., 2015; Gómez Tabares \& Narváez Marín, 2019; Kokkinos \& Kipritsi, 2018; van Noorden et al., 2014), a la vez que promueve la aparición y mantenimiento de todo tipo de comportamientos que van en contravía de los estándares morales a nivel social (Gómez Tabares \& Landinez-Martinez, 2021). De manera particular, los adolescentes que hacen uso de la deshumanización no logran experimentar empatía emocional hacia las personas a las cuales dirigen la agresión (Bandura et al., 1975; Van Noorden et al., 2014). Aspectos notorios al respecto se visualizan en estudios recientes a nivel de jóvenes, violencia y paz, en términos de prácticas cotidianas, especialmente a nivel de sectores populares con insatisfacción de necesidades básicas y con altos índices de violencia y desempleo (Gómez Etayo, Núñez, Giraldo-Rojas et al., 2020; Gómez Tabares, Núñez, Arango Vásquez et al., 2020; Núñez, Arango Vásquez et al., 2020; Núñez, Gallo Consuegra, Gómez Etayo, Carantón Sánchez et al., 2020).

En cuanto al sexo, diversos estudios con adolescentes han reportado que los hombres hacen mayor uso de los mecanismos de desconexión moral, lo cual se ha asociado a una mayor tendencia de problemas de comportamiento disruptivo e intimidación escolar, en comparación con las mujeres (Bjärehed et al., 2019; De Caroli et al., 2011). También se ha encontrado que los adolescentes hombres presentan una mayor orientación a comportamientos externalizantes, entre ellos, agresión física, trasgresión de normas sociales, intimidación, conductas desafiantes y presencia de mayor impulsividad, lo que explicaría la predominancia en el uso de la desconexión moral como estrategia cognitiva para justificar estos comportamientos, mientras que las mujeres presentan una mayor tendencia a conductas internalizantes, entre ellas, quejas somáticas, retraimiento social, baja autoestima, ansiedad y síntomas de depresión (Alarcón Parco \& Bárrig Jó, 2015; Campos et al., 2019; Rescorla et al., 2019).

Estas diferencias en las expresiones comportamentales de hombres y mujeres se han atribuido, en gran parte, a explicaciones de orden socioambiental y cultural, asociadas a la crianza, la socialización, el aprendizaje vicario y directo de patrones divergentes de conducta social según estereotipos por sexo (Gómez Tabares, 2019a; Gómez Etayo, Núñez \& Gallo Consuegra, 2020; Núñez, Gallo Consuegra, Gómez Etayo, Vargas Zuluaga et al., 2020). Sin embargo, no hay estudios que contrasten estos patrones psicológicos y comportamentales entre hombres y mujeres con el uso de las estrategias sociocognitivas de desconexión moral, lo que justifica la importancia de realizar investigaciones en esta dirección. 
Otro aspecto importante por resaltar es la predominancia de estudios psicológicos que han encontrado asociaciones directas y de mediación entre la desconexión moral y todo tipo de conductas disruptivas en la adolescencia, entre ellas, el acoso e intimidación escolar (Bussey et al., 2015; Gini, 2006; Gini et al., 2015; Raskauskas et al., 2010), la delincuencia y las conductas antisociales (D'Urso et al., 2018; Petruccelli et al., 2017), el consumo de alcohol y drogas (Quinn \& Bussey, 2015a, 2015b) y la tendencia a decir mentiras (Doyle \& Bussey, 2018). Sin embargo, no se tienen evidencias concluyentes respecto del efecto que tienen ciertos rasgos clínico-psicológicos en los adolescentes en el uso de los mecanismos de desconexión moral.

El estado actual de la investigación se ha enfocado en explicar cómo la desconexión moral tiene un efecto predictor sobre ciertos tipos de conductas sociales disruptivas de los adolescentes, pero aún se desconoce si existe o no algún tipo de asociación entre indicadores clínico-psicológicos, entre ellos, la ansiedad, la desesperanza, la autoestima, la depresión, la impulsividad y el uso de mecanismos específicos de desconexión moral. En la actualidad, no existen estudios puntuales que analicen las asociaciones entre estos indicadores y la desconexión moral, y mucho menos que examinen divergencias de indicadores clínicos entre hombres y mujeres en cuanto a la predominancia de mecanismos específicos de desconexión moral. En la revisión documental se encontró únicamente un estudio con cierta similitud con las medidas de evaluación de interés, el cual analizó los predictores afectivos, comportamentales y de salud mental en jóvenes involucrados en pandillas (Frisby-Osman \& Wood, 2020). Los análisis revelaron que los jóvenes pandilleros presentaron mayores puntuaciones en ansiedad, problemas de conducta, depresión, rumiación y desconexión moral en comparación con los no pandilleros, pero no se hallaron diferencias de sexo en los grupos en cuanto a estas variables (Frisby-Osman \& Wood, 2020). Sin embargo, en este estudio queda el vacío respecto de si estos factores afectivos, comportamentales y de salud mental inciden en la aparición de la desconexión moral, independientemente de la pertenencia a pandillas.

De acuerdo con lo presentado hasta el momento, es claro el vacío en la investigación y la necesidad de ampliar la evidencia empírica en cuanto a la comprensión de los factores psicológico-clínicos, tanto adaptativos como desadaptativos - y las diferencias en cuanto al sexo-, asociados a la predicción de los diferentes mecanismos específicos de desconexión moral en adolescentes.

La evidencia empírica indica que los adolescentes que presentan comportamientos disruptivos recurren a la desconexión moral como una estrategia para afrontar las emociones negativas de culpa y vergüenza que se desprenden de sus acciones o las consecuencias que genera en los demás (Tillman et al., 2017).

De acuerdo con esta evidencia que indica que la desconexión moral es una estrategia sociocognitiva que se utiliza para evitar el malestar psicológico, este estudio partió de la tesis de que los indicadores clínicopsicológicos que producen malestar emocional, entre ellos, la depresión, la ansiedad, la desesperanza, la impulsividad y una autoestima negativa (autodesprecio), están asociados al uso de los mecanismos desconexión moral. Por el contrario, la autoestima, en términos de una autovaloración positiva, opera como un factor de protección de la desconexión moral. Asimismo, la literatura ha sugerido que estos factores psicológicos presentan diferencias en cuanto al sexo y, por tanto, se complementó la tesis con la idea de que existen efectos divergentes de estos factores psicológicos entre hombres y mujeres.

$\mathrm{Al}$ respecto, en modelos teórico-clínicos, como el de estrés-diátesis (Oquendo et al., 2004), se ha planteado que factores como la depresión, la desesperanza, la impulsividad y la baja autoestima son conducentes a conductas desadaptativas, por ejemplo, agresión auto y hetero dirigida, actos temerarios, déficit en las relaciones interpersonales, ideación e intento de suicidio, consumo de sustancias y, por tanto, son consideradas, entre muchas otras variables, factores de vulnerabilidad psicosocial (Gómez Tabares, Núñez et al., 2019). Sin embargo, la relación de estas características clínicas de vulnerabilidad psicológica conducentes a la desconexión moral en adolescentes aún está por explorarse, lo cual abre múltiples aristas que deben contemplarse como una línea investigativa fructífera en la actualidad. Es importante tener en cuenta que, para determinar con mayor precisión si factores clínicos a nivel individual, tales como la impulsividad, la depresión, la desesperanza, la ansiedad y la autoestima, están asociados a la desconexión moral y sus mecanismos, es necesario un mayor soporte empírico y nuevas aproximaciones investigativas, lo cual aportaría al desarrollo de nuevas comprensiones teóricas en una línea de trabajo que busca generar un puente articulador entre las perspectivas psicológicas clínicas y las cognitivo-sociales, con implicaciones importantes orientadas a la intervención psicosocial con adolescentes y con estrategias integradoras, tal como se ha formulado en poblaciones similares (Núñez Rojas et al., 2006).

Se considera que el análisis de estos factores asociados a la desconexión moral es un insumo altamente valioso para generar estrategias psicoeducativas y psicológicas que estimulen la salud mental, el manejo de 
emociones y criterios morales prosociales en los procesos de acompañamiento psicoeducativo, terapéutico y psicosocial de esta población.

En este sentido, el objetivo de este trabajo fue analizar las diferencias de sexo y predictores clínicos de los mecanismos de desconexión moral en adolescentes escolares. A partir de los resultados obtenidos en investigaciones previas, los vacíos identificados y la conceptualización teórica, se plantearon cuatro hipótesis:

H1: Los hombres presentan puntuaciones más altas que las mujeres en todos los mecanismos de desconexión moral.

H2: La autoconfianza (autoestima positiva) presenta una correlación inversa y el autodesprecio (autoestima negativa), directa, con la desconexión moral total y los ocho mecanismos sociocognitivos.

H3: Las variables clínico-psicológicas de depresión, ansiedad, desesperanza e impulsividad presentan correlaciones directas y efectos predictores de la desconexión moral total y los diferentes mecanismos sociocognitivos.

H4: La impulsividad presenta un mayor efecto predictor de la desconexión moral total y los ocho mecanismos sociocognitivos en los hombres, mientras que la depresión, ansiedad y desesperanza aportan un mayor efecto predictor en las mujeres.

\section{Método}

Es un estudio de enfoque cuantitativo, diseño no experimental de corte transversal. El alcance es explicativo (Hernández-Sampieri et al., 2014).

\section{Participantes}

Participaron 365 adolescentes escolares de dos instituciones educativas privadas de Manizales, Colombia, las cuales fueron elegidas por conveniencia. Se excluyeron 11 adolescentes por no responder todos los instrumentos o dejar preguntas sin responder, por lo que la muestra final estuvo constituida por 354 adolescentes escolares. Para la selección de los adolescentes se utilizó un muestreo probabilístico aleatorio estratificado por sexo y edad. El $57,1 \%(n=202)$ son mujeres y el $42,9 \%(n=152)$ son hombres. La edad media fue de 15,58 ( $D E=1,22)$. Las edades comprendidas fueron de los 14 a los 18 años, diferenciadas de la siguiente manera: 14 años: $22 \%(n=78), 15$ años: $28,2 \%(n=100), 16$ años: $28,5 \%(n=101), 17$ años: $11,6 \%(n=41)$ y 18 años: $9,6 \%(n=34)$.

\section{Instrumentos}

\section{Cuestionario de Mecanismos de Desconexión Moral (Mechanisms of Moral Disengagement Scale - MMDS-; Bandura et al., 1996)}

El cuestionario fue diseñado para evaluar la desconexión moral y el efecto sobre la conducta agresiva y antisocial. Se implementó la versión traducida y validada en España por Rubio-Garay et al. (2017), que consta de 32 ítems tipo Likert con cinco opciones $(1=$ fuertemente en desacuerdo hasta $5=$ totalmente de acuerdo). La escala arroja una puntuación total y ocho puntajes para cada uno de los mecanismos de desconexión moral, con cuatro ítems cada uno: justificación moral (e.g., Está bien pelear por tus amigos), lenguaje eufemístico (e.g., Agredir a alguien es solo un juego), comparación ventajosa (e.g., Dañar alguna propiedad no es gran cosa si se considera que otros hacen cosas peores), desplazamiento de la responsabilidad (e.g., Si las personas viven en malas condiciones no pueden ser culpados por agredir), difusión de la responsabilidad (e.g., Un chico que pertenece a una pandilla no puede ser culpado por los problemas causados por la pandilla), distorsión de las consecuencias (e.g., Decir pequeñas mentiras realmente no hace daño), atribución de la culpa (e.g., Algunas personas merecen ser maltratadas por las cosas que hacen) y deshumanización de la víctima (e.g., Alguien detestable no merece ser tratado como un ser humano). El puntaje total para cada mecanismo se obtiene de promediar el puntaje obtenido en los cuatro ítems.

El análisis de la dimensionalidad y propiedades psicométricas de la versión en español del cuestionario, realizado en una muestra de 513 escolares, evidenció un modelo con un factor general de segundo orden que es compatible con el constructo de desconexión moral e incluye tres dimensiones de primer orden 
(desvinculación por despersonalización, desvinculación por irresponsabilidad, y desvinculación por racionalización), las cuales contienen, a la vez, los ocho mecanismos propuestos originalmente por Bandura et al. (1996). El modelo sugerido presentó un buen ajuste, validez y consistencia interna (alfa de Cronbach) para las tres dimensiones que integran los ocho mecanismos. La versión en español ha evidenciado buenos índices de consistencia interna, estimada con alfa de Cronbach, para la escala total y subescalas, oscilando entre 0,78 a 0,91 en estudios con adolescentes en Colombia (Gómez Tabares, Narváez Marín et al., 2019; Gómez Tabares \& Narváez Marín, 2019). Para este estudio se realizó un análisis de consistencia interna mediante alfa de Cronbach, arrojando un coeficiente de 0,96. La consistencia interna de las subescalas osciló entre 0,66 y 0,81 .

\section{Escala de Impulsividad de Barratt, Versión 11 (Barratt Impulsiveness Scale Version 11 -BIS-11-; Patton et al., 1995)}

Es una de las escalas más utilizadas en el mundo para evaluar la impulsividad. La versión en español consta de 30 preguntas tipo Likert con cuatro opciones de respuesta $(0=$ raramente $o$ nunca hasta $4=$ siempre o casi siempre). La escala ha sido adaptada y validada al español por Oquendo et al. (2001), la cual evidenció una adecuada equivalencia lingüística y conceptual de los ítems, siendo la versión más utilizada en Colombia y Latinoamérica. La escala arroja una puntuación general global y tres subtipos de impulsividad: impulsividad cognitiva (8 ítems) (e.g., Mis pensamientos pueden tener gran velocidad [tengo pensamientos que van muy rápido en mi mente]), impulsividad motora (10 ítems) (e.g., Se me hace difícil estar quieto/a por largos periodos de tiempo) e impulsividad no planeada (12 ítems) (e.g., Planifico mis tareas con cuidado [R]). La puntuación total es la suma de todos los ítems y la de las subescalas, la suma de los correspondientes a cada una de ellas. En estudios con población de niños y jóvenes colombianos, la escala BIS-11 ha reportado índices totales de consistencia interna con alfa de Cronbach de 0,795 (Chahín Pinzón et al., 2019) y de 0,7 (Urrego Barbosa et al., 2017). Las dimensiones de la escala han mostrado correlaciones directas entre sí y validez convergente con las dimensiones del Cuestionario I6 Junior de impulsividad (Chahín Pinzón et al., 2019). Para este estudio se realizó un análisis de consistencia interna, mediante alfa de Cronbach, arrojando un coeficiente de 0,68 para la escala total.

\section{Escala de Desesperanza de Beck (Beck Hopelessness Scale-BHS-; Beck et al., 1974)}

Este instrumento fue diseñado para evaluar las creencias relativas a la desesperanza, el pesimismo, motivación hacia la vida y expectativas hacia el futuro. Es ampliamente utilizada en población de adolescentes y adultos. La versión en español consta de 20 afirmaciones dicotómicas, con opciones de respuesta $0=$ falso y $1=$ verdadero (e.g., No he tenido oportunidades y creo que no las tendré en el futuro). La puntuación total oscila entre 0 a 20 y ofrece un estimado del nivel de desesperanza. La versión en español de la escala ha mostrado buenas propiedades psicométricas y consistencia interna en diversos estudios con población clínica y no clínica, arrojando índices de consistencia interna, evaluada con alfa de Cronbach, entre 0,82 y 0,93 y una confiabilidad test-retest entre 0,60 a 0,69 (González Cifuentes, 2009; Rueda-Jaimes et al., 2018). La validación colombiana con población clínica con riesgo suicida (RuedaJaimes et al., 2018) y no clínica (González Cifuentes, 2009) reporta una adecuada consistencia interna, 0,9 (fórmula 20 de Kuder-Richardson) y 0,83 (alfa de Cronbach), respectivamente. En cuanto a la validez convergente se ha reportado correlaciones directas con el Cuestionario de Comportamiento Suicida Revisado (SBQ-R) $(r h o=0,48, p<0,001)$ (Rueda-Jaimes et al., 2018). El índice de consistencia interna para este estudio fue de 0,78 , el cual se calculó con alfa de Cronbach.

\section{Inventario de Depresión de Beck (Beck Depression Inventory-BDI-; Beck et al., 1979)}

Evalúa la severidad de los síntomas de depresión en diversos grupos poblacionales. Consta de 21 ítems en los que se indagan aspectos cognitivos y somáticos asociados al cuadro clínico. Las opciones de respuesta (e.g., Me siento tan triste o desgraciado que no puedo soportarlo) están ordenadas de menos a mayor gravedad $(0=$ menor gravedad a $3=$ mayor gravedad $)$. Las puntuaciones van de 0 a 63 . Ha sido validado al español por Sanz et al. (2014), arrojando una adecuada sensibilidad y especificidad $(>0,70)$ para discriminar sintomatología depresiva, utilizando los puntos de corte de la versión original. El estudio psicométrico realizado por Sanz y Vázquez (1998) evidenció coeficientes de consistencia interna de 0,83 (alfa de Cronbach) y de confiabilidad test-retest que oscilaban entre 0,60 y 0,972. En cuanto a la validez convergente se ha reportado correlaciones directas con la escala de depresión de Zung y validez 
discriminante respecto a síntomas de ansiedad (Sanz \& Vázquez, 1998). Para Colombia, el inventario ha sido utilizado en diversos estudios con población de adolescentes y adultos, mostrando ser un instrumento con buenos indicadores de consistencia interna, con alfas de Cronbach que oscilan entre 0,86 a 0,90 (Ceballos-Ospino et al., 2015; Gómez Tabares, Núñez, Agudelo Osorio et al., 2020). Para este estudio se realizó un análisis de consistencia interna con alfa de Cronbach, evidenciando un coeficiente de 0,89.

\section{Escala de Autoestima de Rosenberg (Rosenberg Self-Esteem Scale-RSES-; Rosenberg, 1965)}

Evalúa la percepción de autoestima y la valía personal. Se ha implementado en diversos grupos poblacionales. Consta de 10 preguntas tipo Likert de cuatro opciones de respuesta $(1=$ Totalmente en desacuerdo a $4=$ Totalmente de acuerdo). El puntaje total varía de 10 a 40 puntos y establece un estimado general de la autoestima. Las puntuaciones más altas indican buena autoestima. También se puede utilizar un modelo bifactorial, en el cual se evalúa la autoconfianza, compuesta por 5 ítems (autoestima positiva; e.g., Siento que soy una persona que vale tanto como los demás) y el autodesprecio, compuesto por 5 ítems (autoestima negativa; e.g., En general tiendo a pensar que soy un fracaso).

Estudios psicométricos con población colombiana (Cogollo et al., 2015; Gómez-Lugo et al., 2016) han reportado una buena consistencia interna (alfa de Cronbach), oscilando entre 0,72 y 0,86. Al respecto, la versión validada con población colombiana presentó propiedades psicométricas adecuadas con alfa de Cronbach de 0,83 y 0,86 , validez concurrente con correlaciones directas con la escala de autoestima sexual y adecuados indicadores de bondad de ajuste tanto un modelo unifactorial como bifactorial (Gómez-Lugo et al., 2016). Para este estudio se utilizó el modelo bifactorial y el análisis de consistencia interna con alfa de Cronbach evidenció un coeficiente de 0,78.

\section{Inventario de Ansiedad de Beck (Beck Anxiety Inventory-BAI-; Beck et al., 1988)}

Es un inventario autoaplicado para evaluar los síntomas clínicos de ansiedad. Se compone de 21 ítems con cuatro opciones de respuesta $(0=$ en absoluto a $3=$ severamente), en los que se indagan aspectos afectivos-cognitivos (e.g., Miedo a morirme) y somáticos (e.g., Palpitaciones o taquicardia) asociados al cuadro clínico. Los puntajes van de 0 a 63, donde 63 representa el indicador clínico de ansiedad más alto. El análisis psicométrico de la versión en español en población no clínica evidenció una estimación de consistencia interna de 0,93, mediante el coeficiente alfa de Cronbach, y presentó correlaciones $(r=0,63)$ con el cuestionario de depresión BDI (Magán et al., 2008). La consistencia interna para este estudio, evaluada con alfa de Cronbach, fue de 0,915.

\section{Procedimiento}

En consideración con la ley 1090 (Se reglamenta el ejercicio de la profesión de psicología, 2006) y la resolución 8430 (Se establecen las normas científicas, 1993), esta investigación obedeció a los principios éticos de respeto, intimidad y dignidad, asegurando la confidencialidad y el anonimato de los participantes. El estudio contó con aval del comité de ética de la Universidad Católica Luis Amigó para la aplicación de los instrumentos y la firma del consentimiento informado de los padres de familia y el asentimiento informado de los participantes. La aplicación de los instrumentos se realizó en las aulas de clase entre los meses de marzo a mayo del 2019 y fueron aplicados por el investigador principal. Se inició con el cuestionario de mecanismos de desconexión moral y posteriormente los instrumentos de autoestima, impulsividad, desesperanza, ansiedad y depresión.

\section{Análisis de Datos}

Los resultados de la aplicación de los instrumentos de medición fueron digitalizados y codificados en una matriz de datos de Excel. Inicialmente se hizo una revisión de la base de datos, donde se aseguró que no hubiera datos perdidos o respuestas que no se ajustaran a las opciones de los instrumentos. Para el análisis se utilizó el paquete estadístico SPSS v. 25. Se aplicó el coeficiente de alfa de Cronbach para estimar la consistencia interna de las escalas y subescalas y la prueba Kolmogorov-Smirnov para determinar la normalidad. Se procedió a realizar un análisis comparativo de los mecanismos de desconexión moral en función del sexo, utilizando la prueba $t$ de Student para muestras independientes. El tamaño del efecto ( $d$ de Cohen) se calculó en $R$ Studio Cloud. Posteriormente, se realizó un análisis de correlación, utilizando el coeficiente $r$ de Pearson. Se estimó un modelo general de regresión lineal múltiple, 
mediante el método de introducción, en el que se utilizaron los mecanismos de desconexión moral como variables de respuesta, con el fin de detectar los factores con mayor efecto predictor sobre la desconexión moral total y cada uno de los mecanismos en los adolescentes estudiados. La autoconfianza, autodesprecio, impulsividad, desesperanza, ansiedad, depresión y sexo se ubicaron como las variables predictoras y la desconexión moral total y los ocho mecanismos como las variables dependientes. Finalmente, se estimó un modelo de regresión, tanto para hombres como mujeres, con el fin de analizar los efectos de las variables predictores según el sexo.

\section{Resultados}

En la Tabla 1 se muestra el análisis comparativo de los mecanismos de desconexión moral en función del sexo, evidenciando que los hombres presentan puntuaciones más altas que las mujeres en todas las variables de estudio $(p<0,001)$, con tamaños del efecto intermedios $(d \geq 0,5$; Cohen, 1988), salvo en difusión de la responsabilidad, lo que indica que las diferencias son independientes del tamaño de la muestra.

Tabla 1

Análisis Comparativo de los Mecanismos de Desconexión Moral en Hombres y Mujeres

\begin{tabular}{lccccccc}
\hline \multirow{2}{*}{\multicolumn{1}{c}{ Variable }} & \multicolumn{2}{c}{ Mujer $(n=202)$} & \multicolumn{2}{c}{ Hombre $(n=152)$} & \multicolumn{2}{c}{ Estadístico de prueba } \\
\cline { 2 - 8 } & $M$ & $D E$ & $M$ & $D E$ & $t$ & $p$ & $d$ \\
\hline Desconexión moral total & 1,56 & 0,48 & 1,96 & 0,84 & 5,529 & $<0,001$ & 0,6 \\
Justificación moral & & & & & & & \\
Lenguaje eufemístico & 1,63 & 0,67 & 2,19 & 1,00 & 6,238 & $<0,001$ & 0,7 \\
Comparación ventajosa & 1,68 & 0,68 & 2,15 & 0,95 & 5,428 & $<0,001$ & 0,6 \\
Desplazamiento de la responsabilidad & 1,35 & 0,53 & 1,65 & 0,86 & 4,075 & $<0,001$ & 0,4 \\
Difusión de la responsabilidad & 1,63 & 0,61 & 1,93 & 0,91 & 3,659 & $<0,001$ & 0,4 \\
Distorsión de las consecuencias & 1,65 & 0,66 & 1,96 & 0,92 & 3,231 & $<0,001$ & 0,3 \\
Atribución de la culpa & 1,47 & 0,60 & 1,83 & 0,94 & 4,397 & $<0,001$ & 0,6 \\
Deshumanización & 1,41 & 0,62 & 1,83 & 1,00 & 4,837 & $<0,001$ & 0,5 \\
\hline
\end{tabular}

En la Tabla 2 se muestra el análisis de correlaciones entre las variables clínico-psicológicas, la desconexión moral total y los mecanismos específicos. Se encontró que las variables impulsividad, desesperanza y ansiedad presentaron correlaciones directas $(p<0,001)$ con la desconexión moral total y los ocho mecanismos sociocognitivos. La depresión se correlacionó directamente $(p<0,05)$ con el lenguaje eufemístico, el desplazamiento de responsabilidad y la distorsión de consecuencias. Con excepción de la comparación ventajosa y la deshumanización, el autodesprecio (autoestima negativa) se correlacionó directamente con los diferentes mecanismos de desconexión moral $(p<0,05)$. Finalmente, la autoconfianza (autoestima positiva) se correlacionó inversamente $(p<0,05)$ con la desconexión moral total y los mecanismos de justificación moral, lenguaje eufemístico, difusión de responsabilidad, distorsión de las consecuencias y atribución de la culpa.

En la Tabla 3 se muestran los efectos de las variables independientes sobre la desconexión moral y los mecanismos. Del total de variables predictoras, la impulsividad, la desesperanza, la ansiedad y el sexo presentaron un efecto significativo, explicando el $22 \%$ de la variación de la desconexión moral total.

En cuanto a los mecanismos desconexión moral, la impulsividad, la desesperanza, la ansiedad y el sexo fueron las variables que aportaron un efecto significativo que explica el $24 \%$ de la variación del mecanismo de justificación moral, mientras que la impulsividad, desesperanza y sexo presentaron un efecto que logra explicar el 18\% de los mecanismos de lenguaje eufemístico y distorsión de las consecuencias. La impulsividad, ansiedad y sexo fueron las variables que aportaron un efecto que explica el $14 \%$ del desplazamiento de responsabilidad y el $17 \%$ de la atribución de la culpa. La impulsividad y el sexo aportaron los efectos que logran explicar el 15\% de la variación de la comparación ventajosa y el 11\% de la difusión de la responsabilidad. La deshumanización es explicada en un 19\% por el efecto de la impulsividad, 
desesperanza, ansiedad y sexo. La impulsividad y el sexo son los predictores presentes en cada uno de mecanismos de desconexión moral.

Tabla 2

Correlaciones entre los Mecanismos de Desconexión Moral y las Variables Clínico-Psicológicas

\begin{tabular}{|c|c|c|c|c|c|c|}
\hline Variable & Autoconfianza & Autodesprecio & Impulsividad & Desesperanza & Ansiedad & Depresión \\
\hline Desconexión moral total & $-0,125^{*}$ & $0,142^{* *}$ & $0,347^{* *}$ & $0,209^{* *}$ & $0,210^{* *}$ & 0,100 \\
\hline Justificación moral & $-0,124^{*}$ & $0,146^{* *}$ & $0,336^{* *}$ & $0,224^{* *}$ & $0,210^{* *}$ & 0,077 \\
\hline Lenguaje eufemístico & $-0,111^{*}$ & $0,121^{*}$ & $0,300^{* *}$ & $0,181^{* *}$ & $0,160^{* *}$ & $0,107^{*}$ \\
\hline Comparación ventajosa & $-0,073$ & 0,096 & $0,294^{* *}$ & $0,168^{* *}$ & $0,169^{* *}$ & 0,038 \\
\hline Desplazamiento de responsabilidad & $-0,099$ & $0,122^{*}$ & $0,280^{* *}$ & $0,149^{* *}$ & $0,183^{* *}$ & $0,132^{*}$ \\
\hline Difusión de responsabilidad & $-0,110^{*}$ & $0,123^{*}$ & $0,255^{* *}$ & $0,147^{* *}$ & $0,179^{* *}$ & 0,096 \\
\hline Distorsión de consecuencias & $-0,118^{*}$ & $0,138^{* *}$ & $0,290^{* *}$ & $0,184^{* *}$ & $0,151^{* *}$ & $0,126^{*}$ \\
\hline Atribución de la culpa & $-0,121^{*}$ & $0,130^{*}$ & $0,312^{* *}$ & $0,160^{* *}$ & $0,207^{* *}$ & 0,058 \\
\hline Deshumanización & $-0,100$ & 0,096 & $0,310^{* *}$ & $0,214^{* *}$ & $0,183^{* *}$ & 0,047 \\
\hline
\end{tabular}

$* * p=0,01 ; * p=0,05$.

Tabla 3

Efecto de las Variables Predictoras sobre los Mecanismos de Desconexión Moral

\begin{tabular}{|c|c|c|c|c|c|c|c|}
\hline Variable dependiente & Predictor & $B$ & $E E$ & $\beta$ & $t$ & $p$ & $95 \%$ IC para $B$ \\
\hline \multirow[t]{7}{*}{ Desconexión moral } & Autoconfianza & 0,026 & 0,153 & 0,018 & 0,169 & 0,866 & $-0,275,0,327$ \\
\hline & Autodesprecio & 0,018 & 0,105 & 0,017 & 0,169 & 0,866 & $-0,190,0,225$ \\
\hline & Impulsividad & 0,478 & 0,091 & 0,289 & 5,257 & $<0,001$ & $0,299,0,656$ \\
\hline & Desesperanza & 0,050 & 0,022 & 0,113 & 2,313 & 0,021 & $0,008,0,093$ \\
\hline & Ansiedad & 0,006 & 0,003 & 0,115 & 2,129 & 0,034 & $0,000,0,012$ \\
\hline & Depresión & $-0,001$ & 0,006 & $-0,009$ & $-0,146$ & 0,884 & $-0,012,0,010$ \\
\hline & Sexo & $-0,373$ & 0,069 & $-0,269$ & $-5,431$ & $<0,001$ & $-0,508,-0,238$ \\
\hline \multicolumn{8}{|c|}{$F(7,346)=14,194, p<0,001, R^{2}=0,223$} \\
\hline \multirow{7}{*}{ Justificación moral } & Autoconfianza & 0,023 & 0,192 & 0,013 & 0,120 & 0,904 & $-0,355,0,401$ \\
\hline & Autodesprecio & 0,031 & 0,132 & 0,023 & 0,233 & 0,816 & $-0,230,0,291$ \\
\hline & Impulsividad & 0,584 & 0,114 & 0,278 & 5,118 & $<0,001$ & $0,359,0,808$ \\
\hline & Desesperanza & 0,070 & 0,027 & 0,124 & 2,564 & 0,011 & $0,016,0,124$ \\
\hline & Ansiedad & 0,009 & 0,004 & 0,124 & 2,322 & 0,021 & $0,001,0,016$ \\
\hline & Depresión & $-0,004$ & 0,007 & $-0,031$ & $-0,513$ & 0,608 & $-0,018,0,010$ \\
\hline & Sexo & $-0,523$ & 0,086 & $-0,297$ & $-6,065$ & $<0,001$ & $-0,693,-0,354$ \\
\hline \multicolumn{8}{|c|}{$F(7,346)=15,635, p<0,001, R^{2}=0,240$} \\
\hline \multirow[t]{7}{*}{ Lenguaje eufemístico } & Autoconfianza & 0,024 & 0,191 & 0,014 & 0,125 & 0,901 & $-0,353,0,400$ \\
\hline & Autodesprecio & 0,009 & 0,132 & 0,007 & 0,071 & 0,943 & $-0,250,0,269$ \\
\hline & Impulsividad & 0,493 & 0,114 & 0,245 & 4,339 & $<0,001$ & $0,270,0,717$ \\
\hline & Desesperanza & 0,057 & 0,027 & 0,105 & 2,126 & 0,034 & $0,004,0,109$ \\
\hline & Ansiedad & 0,005 & 0,004 & 0,069 & 1,251 & 0,212 & $-0,003,0,012$ \\
\hline & Depresión & 0,004 & 0,007 & 0,037 & 0,595 & 0,552 & $-0,010,0,018$ \\
\hline & Sexo & $-0,462$ & 0,086 & $-0,273$ & $-5,372$ & $<0,001$ & $-0,631,-0,293$ \\
\hline \multicolumn{8}{|c|}{$F(7,346)=10,901, p<0,001, R^{2}=0,181$} \\
\hline
\end{tabular}

(continúa) 
Tabla 3 (Conclusión)

Efecto de las Variables Predictoras sobre los Mecanismos de Desconexión Moral

\begin{tabular}{|c|c|c|c|c|c|c|c|}
\hline Variable dependiente & Predictor & $B$ & $E E$ & $\beta$ & $t$ & $p$ & $95 \%$ IC para $B$ \\
\hline \multirow[t]{7}{*}{ Comparación ventajosa } & Autoconfianza & 0,053 & 0,163 & 0,036 & 0,323 & 0,747 & $-0,269,0,374$ \\
\hline & Autodesprecio & 0,019 & 0,113 & 0,018 & 0,170 & 0,865 & $-0,202,0,241$ \\
\hline & Impulsividad & 0,463 & 0,097 & 0,274 & 4,774 & $<0,001$ & $0,272,0,654$ \\
\hline & Desesperanza & 0,043 & 0,023 & 0,094 & 1,846 & 0,066 & $-0,003,0,089$ \\
\hline & Ansiedad & 0,006 & 0,003 & 0,101 & 1,789 & 0,075 & $-0,001,0,012$ \\
\hline & Depresión & $-0,006$ & 0,006 & $-0,066$ & $-1,052$ & 0,294 & $-0,018,0,006$ \\
\hline & Sexo & $-0,266$ & 0,073 & $-0,187$ & $-3,621$ & $<0,001$ & $-0,410,-0,121$ \\
\hline \multicolumn{8}{|c|}{$F(7,346)=8,721, p<0,001, R^{2}=0,150$} \\
\hline \multirow{7}{*}{$\begin{array}{l}\text { Desplazamiento de la } \\
\text { responsabilidad }\end{array}$} & Autoconfianza & 0,184 & 0,179 & 0,116 & 1,026 & 0,306 & $-0,169,0,537$ \\
\hline & Autodesprecio & 0,105 & 0,124 & 0,091 & 0,850 & 0,396 & $-0,138,0,348$ \\
\hline & Impulsividad & 0,393 & 0,107 & 0,214 & 3,687 & $<0,001$ & $0,183,0,602$ \\
\hline & Desesperanza & 0,036 & 0,026 & 0,072 & 1,395 & 0,164 & $-0,015,0,086$ \\
\hline & Ansiedad & 0,007 & 0,003 & 0,113 & 2,078 & 0,038 & $0,000,0,014$ \\
\hline & Depresión & 0,007 & 0,007 & 0,071 & 1,109 & 0,268 & $-0,006,0,020$ \\
\hline & Sexo & $-0,299$ & 0,081 & $-0,194$ & $-3,706$ & $<0,001$ & $-0,457,-0,140$ \\
\hline \multicolumn{8}{|c|}{$F(7,346)=7,660, p<0,001, R^{2}=0,143$} \\
\hline \multirow{7}{*}{$\begin{array}{l}\text { Difusión de la } \\
\text { responsabilidad }\end{array}$} & Autoconfianza & 0,045 & 0,188 & 0,027 & 0,238 & 0,812 & $-0,325,0,414$ \\
\hline & Autodesprecio & 0,045 & 0,129 & 0,038 & 0,350 & 0,726 & $-0,209,0,300$ \\
\hline & Impulsividad & 0,374 & 0,111 & 0,197 & 3,356 & 0,001 & $0,155,0,593$ \\
\hline & Desesperanza & 0,040 & 0,027 & 0,078 & 1,496 & 0,135 & $-0,013,0,093$ \\
\hline & Ansiedad & 0,007 & 0,004 & 0,104 & 1,810 & 0,071 & $-0,001,0,014$ \\
\hline & Depresión & 0,001 & 0,007 & 0,007 & 0,105 & 0,917 & $-0,013,0,014$ \\
\hline & Sexo & $-0,262$ & 0,084 & $-0,164$ & $-3,101$ & 0,002 & $-0,427,-0,096$ \\
\hline \multicolumn{8}{|c|}{$F(7,346)=3,479, p<0,001, R^{2}=0,111$} \\
\hline \multirow{7}{*}{$\begin{array}{l}\text { Distorsión de las } \\
\text { consecuencias }\end{array}$} & Autoconfianza & 0,116 & 0,188 & 0,068 & 0,616 & 0,539 & $-0,254,0,485$ \\
\hline & Autodesprecio & 0,087 & 0,129 & 0,069 & 0,669 & 0,504 & $-0,168,0,341$ \\
\hline & Impulsividad & 0,443 & 0,112 & 0,224 & 3,968 & $<0,001$ & $0,223,0,662$ \\
\hline & Desesperanza & 0,057 & 0,026 & 0,106 & 2,141 & 0,033 & $0,005,0,109$ \\
\hline & Ansiedad & 0,004 & 0,004 & 0,052 & 0,949 & 0,343 & $-0,004,0,011$ \\
\hline & Depresión & 0,008 & 0,007 & 0,076 & 1,226 & 0,221 & $-0,005,0,022$ \\
\hline & Sexo & $-0,464$ & 0,084 & $-0,280$ & $-5,500$ & $<0,001$ & $-0,630,-0,298$ \\
\hline \multicolumn{8}{|c|}{$F(7,346)=10,866, p<0,001, R^{2}=0,180$} \\
\hline \multirow[t]{7}{*}{ Atribución de la culpa } & Autoconfianza & $-0,067$ & 0,180 & $-0,041$ & $-0,372$ & 0,710 & $-0,421,0,287$ \\
\hline & Autodesprecio & $-0,022$ & 0,124 & $-0,019$ & $-0,179$ & 0,858 & $-0,267,0,222$ \\
\hline & Impulsividad & 0,510 & 0,107 & 0,271 & 4,766 & $<0,001$ & $0,299,0,720$ \\
\hline & Desesperanza & 0,039 & 0,026 & 0,077 & 1,521 & 0,129 & $-0,011,0,090$ \\
\hline & Ansiedad & 0,009 & 0,004 & 0,134 & 2,411 & 0,016 & $0,002,0,016$ \\
\hline & Depresión & $-0,008$ & 0,007 & $-0,076$ & $-1,220$ & 0,223 & $-0,021,0,005$ \\
\hline & Sexo & $-0,335$ & 0,081 & $-0,212$ & $-4,141$ & $<0,001$ & $-0,494,-0,176$ \\
\hline \multicolumn{8}{|c|}{$F(7,346)=10,190, p<0,001, R^{2}=0,171$} \\
\hline \multirow[t]{7}{*}{ Deshumanización } & Autoconfianza & $-0,170$ & 0,189 & $-0,099$ & $-0,899$ & 0,369 & $-0,542,0,202$ \\
\hline & Autodesprecio & $-0,132$ & 0,130 & $-0,105$ & $-1,012$ & 0,312 & $-0,388,0,124$ \\
\hline & Impulsividad & 0,560 & 0,112 & 0,281 & 4,993 & $<0,001$ & $0,340,0,781$ \\
\hline & Desesperanza & 0,073 & 0,027 & 0,136 & 2,728 & 0,007 & $0,020,0,126$ \\
\hline & Ansiedad & 0,007 & 0,004 & 0,108 & 1,970 & 0,050 & $0,000,0,015$ \\
\hline & Depresión & $-0,009$ & 0,007 & $-0,082$ & $-1,327$ & 0,185 & $-0,023,0,004$ \\
\hline & Sexo & $-0,375$ & 0,085 & $-0,224$ & $-4,419$ & $<0,001$ & $-0,542,-0,208$ \\
\hline \multicolumn{8}{|c|}{$F(7,346)=11,428, p<0,001, R^{2}=0,188$} \\
\hline
\end{tabular}

En la Tabla 4 se muestran los efectos de los factores clínico-psicológicos en hombres y mujeres para el uso de los mecanismos de desconexión moral. En el caso de los hombres, se encontró que, del conjunto de las 
variables predictoras, la impulsividad fue la que aportó un efecto que explica en un $12 \%$ la variación de la desconexión moral total y, en promedio, un $10 \%$ del uso de cada uno de los mecanismos de desconexión moral. De manera particular, la deshumanización es explicada en un 17\% por el efecto de la impulsividad y la desesperanza.

En el caso de las mujeres, la impulsividad también aportó un efecto en la predicción de la desconexión moral total y de los ocho mecanismos. La impulsividad, desesperanza y ansiedad aportaron los efectos que explican el $29 \%$ de la desconexión moral total y el $25 \%$ de la justificación moral, entre tanto, a impulsividad y la ansiedad aportaron los efectos que explican el $17 \%$ del lenguaje eufemístico y el $22 \%$ de la atribución de la culpa. La desesperanza y la impulsividad aportaron los efectos que explican el $18 \%$ de la comparación ventajosa y esta última (impulsividad), junto con la depresión explican el $17 \%$ del desplazamiento de responsabilidad. La impulsividad aportó el único efecto que explica el $12 \%$ de la difusión de responsabilidad. El mecanismo de distorsión de las consecuencias es explicado en un 18\% por las variables impulsividad, desesperanza y depresión, mientras que la impulsividad y desesperanza aportaron un efecto directo y la autoconfianza, un efecto inverso, que explican en un $22 \%$ el mecanismo de deshumanización.

\section{Tabla 4}

Efecto de las Variables Predictoras sobre los Mecanismos de Desconexión Moral en Función del Sexo

\begin{tabular}{|c|c|c|c|c|c|c|c|c|c|}
\hline \multirow{2}{*}{$\begin{array}{c}\text { Variable } \\
\text { dependiente }\end{array}$} & \multirow{2}{*}{ Predictor } & \multicolumn{4}{|c|}{ Modelo Hombres } & \multicolumn{4}{|c|}{ Modelo Mujeres } \\
\hline & & $\beta$ & $t$ & $p$ & $95 \%$ IC para $\beta$ & $\beta$ & $t$ & $p$ & $95 \%$ IC para $\beta$ \\
\hline \multirow[t]{7}{*}{ Desconexión moral } & Autoconfianza & 0,162 & 0,988 & 0,325 & $-0,320,0,960$ & $-0,117$ & $-0,804$ & 0,423 & $-0,375,0,158$ \\
\hline & Autodesprecio & 0,100 & 0,623 & 0,535 & $-0,292,0,560$ & $-0,039$ & $-0,296$ & 0,768 & $-0,214,0,158$ \\
\hline & Impulsividad & 0,267 & 3,081 & 0,002 & $0,205,0,940$ & 0,330 & 4,532 & $<0,001$ & $0,207,0,527$ \\
\hline & Desesperanza & 0,125 & 1,535 & 0,127 & $-0,022,0,172$ & 0,150 & 2,445 & 0,015 & $0,009,0,081$ \\
\hline & Ansiedad & 0,090 & 1,032 & 0,304 & $-0,006,0,018$ & 0,163 & 2,324 & 0,021 & $0,001,0,012$ \\
\hline & Depresión & $-0,078$ & $-0,925$ & 0,356 & $-0,036,0,013$ & 0,055 & 0,669 & 0,505 & $-0,006,0,013$ \\
\hline & & \multicolumn{4}{|c|}{$F(6,145)=3,237, p=0,005, R^{2}=0,118$} & \multicolumn{4}{|c|}{$F(6,195)=13,507, p<0,001, R^{2}=0,294$} \\
\hline \multirow[t]{7}{*}{ Justificación moral } & Autoconfianza & 0,001 & 0,004 & 0,997 & $-0,763,0,766$ & 0,065 & 0,434 & 0,665 & $-0,297,0,465$ \\
\hline & Autodesprecio & $-0,076$ & $-0,471$ & 0,638 & $-0,630,0,387$ & 0,169 & 1,234 & 0,219 & $-0,099,0,432$ \\
\hline & Impulsividad & 0,269 & 3,107 & 0,002 & $0,251,1,129$ & 0,310 & 4,136 & $<0,001$ & $0,251,0,708$ \\
\hline & Desesperanza & 0,124 & 1,512 & 0,133 & $-0,027,0,205$ & 0,153 & 2,428 & 0,016 & $0,012,0,116$ \\
\hline & Ansiedad & 0,100 & 1,140 & 0,256 & $-0,006,0,022$ & 0,181 & 2,519 & 0,013 & $0,002,0,018$ \\
\hline & Depresión & $-0,045$ & $-0,529$ & 0,597 & $-0,037,0,021$ & $-0,022$ & $-0,259$ & 0,796 & $-0,016,0,012$ \\
\hline & & \multicolumn{4}{|c|}{$F(6,145)=3,122, p=0,007, R^{2}=0,114$} & \multicolumn{4}{|c|}{$F(6,195)=10,953, p<0,001, R^{2}=0,252$} \\
\hline \multirow{7}{*}{$\begin{array}{l}\text { Lenguaje } \\
\text { eufemístico }\end{array}$} & Autoconfianza & 0,157 & 0,950 & 0,344 & $-0,380,1,083$ & $-0,094$ & $-0,596$ & 0,552 & $-0,527,0,282$ \\
\hline & Autodesprecio & 0,148 & 0,909 & 0,365 & $-0,263,0,711$ & $-0,099$ & $-0,689$ & 0,492 & $-0,380,0,183$ \\
\hline & Impulsividad & 0,242 & 2,762 & 0,006 & $0,167,1,007$ & 0,250 & 3,171 & 0,002 & $0,147,0,632$ \\
\hline & Desesperanza & 0,147 & 1,780 & 0,077 & $-0,011,0,211$ & 0,072 & 1,089 & 0,277 & $-0,025,0,085$ \\
\hline & Ansiedad & 0,021 & 0,236 & 0,814 & $-0,012,0,015$ & 0,165 & 2,325 & 0,118 & $-0,002,0,015$ \\
\hline & Depresión & $-0,059$ & $-0,690$ & 0,491 & $-0,038,0,018$ & 0,113 & 1,259 & 0,210 & $-0,005,0,024$ \\
\hline & & \multicolumn{4}{|c|}{$F(6,145)=2,670, p=0,017, R^{2}=0,099$} & \multicolumn{4}{|c|}{$F(6,195)=6,743, p<0,001, R^{2}=0,172$} \\
\hline \multirow{7}{*}{$\begin{array}{l}\text { Comparación } \\
\text { ventajosa }\end{array}$} & Autoconfianza & 0,092 & 0,558 & 0,578 & $-0,474,0,846$ & 0,029 & 0,186 & 0,853 & $-0,283,0,342$ \\
\hline & Autodesprecio & 0,018 & 0,111 & 0,912 & $-0,414,0,464$ & 0,072 & 0,507 & 0,613 & $-0,162,0,274$ \\
\hline & Impulsividad & 0,251 & 2,858 & 0,005 & $0,169,0,927$ & 0,301 & 3,845 & $<0,001$ & $0,178,0,553$ \\
\hline & Desesperanza & 0,070 & 0,848 & 0,398 & $-0,057,0,143$ & 0,154 & 2,339 & 0,020 & $0,008,0,093$ \\
\hline & Ansiedad & 0,096 & 1,093 & 0,276 & $-0,005,0,019$ & 0,106 & 1,414 & 0,159 & $-0,002,0,011$ \\
\hline & Depresión & $-0,137$ & $-1,592$ & 0,113 & $-0,046,0,005$ & 0,008 & 0,094 & 0,925 & $-0,011,0,012$ \\
\hline & & \multicolumn{4}{|c|}{$F(6,145)=2,537, p=0,023, R^{2}=0,095$} & \multicolumn{4}{|c|}{$F(6,195)=7,338, p<0,001, R^{2}=0,184$} \\
\hline \multirow{7}{*}{$\begin{array}{l}\text { Desplazamiento de } \\
\text { la responsabilidad }\end{array}$} & Autoconfianza & 0,224 & 1,339 & 0,183 & $-0,228,1,184$ & 0,030 & 0,190 & 0,850 & $-0,329,0,400$ \\
\hline & Autodesprecio & 0,179 & 1,087 & 0,279 & $-0,211,0,728$ & 0,028 & 0,193 & 0,847 & $-0,229,0,279$ \\
\hline & Impulsividad & 0,194 & 2,196 & 0,030 & $0,045,0,855$ & 0,226 & 2,861 & 0,005 & $0,098,0,535$ \\
\hline & Desesperanza & 0,068 & 0,816 & 0,416 & $-0,063,0,151$ & 0,103 & 1,541 & 0,125 & $-0,011,0,088$ \\
\hline & Ansiedad & 0,095 & 1,063 & 0,289 & $-0,006,0,020$ & 0,093 & 1,229 & 0,220 & $-0,003,0,012$ \\
\hline & Depresión & $-0,019$ & $-0,224$ & 0,823 & $-0,030,0,024$ & 0,187 & 2,483 & 0,014 & $0,003,0,025$ \\
\hline & & \multicolumn{4}{|c|}{$F(6,145)=2,230, p=0,046, R^{2}=0,077$} & \multicolumn{4}{|c|}{$F(6,195)=6,526, p<0,001, R^{2}=0,167$} \\
\hline
\end{tabular}


Tabla 4 (Conclusión)

Efecto de las Variables Predictoras sobre los Mecanismos de Desconexión Moral en Función del Sexo

\begin{tabular}{|c|c|c|c|c|c|c|c|c|c|}
\hline \multirow{2}{*}{$\begin{array}{c}\text { Variable } \\
\text { dependiente }\end{array}$} & \multirow{2}{*}{ Predictor } & \multicolumn{4}{|c|}{ Modelo Hombres } & \multicolumn{4}{|c|}{ Modelo Mujeres } \\
\hline & & $\beta$ & $t$ & $p$ & $95 \%$ IC para $\beta$ & $\beta$ & $t$ & $p$ & $95 \%$ IC para $\beta$ \\
\hline \multirow{7}{*}{$\begin{array}{l}\text { Difusión de la } \\
\text { responsabilidad }\end{array}$} & Autoconfianza & 0,174 & 1,041 & 0,300 & $-0,338,1,089$ & $-0,101$ & $-0,617$ & 0,538 & $-0,530,0,278$ \\
\hline & Autodesprecio & 0,113 & 0,686 & 0,494 & $-0,310,0,639$ & $-0,015$ & $-0,100$ & 0,921 & $-0,296,0,267$ \\
\hline & Impulsividad & 0,192 & 2,173 & 0,031 & $0,041,0,859$ & 0,186 & 2,279 & 0,024 & $0,038,0,522$ \\
\hline & Desesperanza & 0,130 & 1,561 & 0,121 & $-0,023,0,193$ & 0,056 & 0,817 & 0,415 & $-0,032,0,078$ \\
\hline & Ansiedad & 0,083 & 0,939 & 0,349 & $-0,007,0,019$ & 0,140 & 1,787 & 0,076 & $-0,001,0,016$ \\
\hline & Depresión & $-0,015$ & $-0,176$ & 0,860 & $-0,030,0,025$ & 0,007 & 0,076 & 0,939 & $-0,014,0,015$ \\
\hline & & \multicolumn{4}{|c|}{$F(6,145)=2,165, p=0,049, R^{2}=0,082$} & \multicolumn{4}{|c|}{$F(6,195)=4,245, p<0,001, R^{2}=0,116$} \\
\hline \multirow{7}{*}{$\begin{array}{l}\text { Distorsión de las } \\
\text { consecuencias }\end{array}$} & Autoconfianza & 0,147 & 0,880 & 0,380 & $-0,412,1,073$ & $-0,013$ & $-0,083$ & 0,934 & $-0,394,0,362$ \\
\hline & Autodesprecio & 0,091 & 0,555 & 0,580 & $-0,355,0,632$ & 0,070 & 0,489 & 0,625 & $-0,198,0,329$ \\
\hline & Impulsividad & 0,249 & 2,831 & 0,005 & $0,184,1,036$ & 0,199 & 2,537 & 0,012 & $0,065,0,518$ \\
\hline & Desesperanza & 0,071 & 0,857 & 0,393 & $-0,064,0,161$ & 0,147 & 2,220 & 0,028 & $0,006,0,109$ \\
\hline & Ansiedad & 0,039 & 0,439 & 0,661 & $-0,011,0,017$ & 0,073 & 0,966 & 0,335 & $-0,004,0,012$ \\
\hline & Depresión & 0,018 & 0,213 & 0,832 & $-0,025,0,032$ & 0,185 & 2,483 & 0,014 & $0,003,0,026$ \\
\hline & & \multicolumn{4}{|c|}{$F(6,145)=2,261, p=0,041, R^{2}=0,086$} & \multicolumn{4}{|c|}{$F(6,195)=7,061, p<0,001, R^{2}=0,178$} \\
\hline \multirow{7}{*}{$\begin{array}{l}\text { Atribución de la } \\
\text { culpa }\end{array}$} & Autoconfianza & 0,133 & 0,802 & 0,424 & $-0,431,1,021$ & $-0,209$ & $-1,362$ & 0,175 & $-0,5810,106$ \\
\hline & Autodesprecio & 0,137 & 0,841 & 0,402 & $-0,278,0,688$ & $-0,156$ & $-1,123$ & 0,263 & $-0,376,0,103$ \\
\hline & Impulsividad & 0,242 & 2,770 & 0,006 & $0,167,1,001$ & 0,305 & 3,993 & $<0,001$ & $0,211,0,623$ \\
\hline & Desesperanza & 0,085 & 1,034 & 0,303 & $-0,052,0,168$ & 0,101 & 1,570 & 0,118 & $-0,010,0,084$ \\
\hline & Ansiedad & 0,084 & 0,953 & 0,342 & $-0,007,0,020$ & 0,195 & 2,657 & 0,009 & $0,002,0,017$ \\
\hline & Depresión & $-0,148$ & $-1,729$ & 0,086 & $-0,052,0,003$ & $-0,029$ & $-0,331$ & 0,741 & $-0,015,0,010$ \\
\hline & & \multicolumn{4}{|c|}{$F(6,145)=2,751, p=0,015, R^{2}=0,102$} & \multicolumn{4}{|c|}{$F(6,195)=9,253, p<0,001, R^{2}=0,222$} \\
\hline \multirow[t]{7}{*}{ Deshumanización } & Autoconfianza & 0,165 & 1,934 & 0,055 & $-0,009,0,852$ & $-0,237$ & $-2,878$ & 0,004 & $-0,471,-0,088$ \\
\hline & Autodesprecio & 0,112 & 0,710 & 0,479 & $-0,319,0,676$ & $-0,047$ & $-0,620$ & 0,536 & $-0,177,0,092$ \\
\hline & Impulsividad & 0,258 & 3,047 & 0,003 & $0,233,1,091$ & 0,284 & 3,710 & $<0,001$ & $0,188,0,616$ \\
\hline & Desesperanza & 0,189 & 2,358 & 0,020 & $0,022,0,249$ & 0,154 & 2,395 & 0,018 & $0,010,0,108$ \\
\hline & Ansiedad & 0,123 & 1,444 & 0,151 & $-0,004,0,024$ & 0,101 & 1,375 & 0,171 & $-0,002,0,013$ \\
\hline & Depresión & $-0,155$ & $-1,865$ & 0,064 & $-0,056,0,002$ & $-0,038$ & $-0,435$ & 0,664 & $-0,016,0,010$ \\
\hline & & \multicolumn{4}{|c|}{$F(6,145)=4,213, p<0,001, R^{2}=0,166$} & \multicolumn{4}{|c|}{$F(6,195)=9,157, p<0,001, R^{2}=0,220$} \\
\hline
\end{tabular}

\section{Discusión}

Al considerar el objetivo principal del estudio, se aprobó la primera hipótesis (H1), dado que se encontró que los hombres presentaron puntuaciones más altas en comparación a las mujeres en cada uno de los mecanismos de desconexión moral. Este hallazgo es coherente con estudios similares con escolares italianos y suecos (Bjärehed et al., 2019; De Caroli et al., 2011; Thornberg \& Jungert, 2014), al encontrar que los hombres hacen mayor uso de la desconexión moral que las mujeres para justificar y legitimar acciones que van en contravía a las normas establecidas. Una razón de esto, y en coherencia con diversos estudios (Correa Duque, 2017; Côté et al., 2002; Gómez Tabares \& Durán Palacio, 2020; Mestre et al., 2009; Redondo Pacheco et al., 2015; Van der Graaff et al., 2014; Van der Graaff et al., 2018), es que los niños presentan una mayor tendencia a la agresión, la impulsividad y la externalización, mientras que las niñas, a la solidaridad, la empatía y los comportamientos prosociales, lo que estaría asociado a una mayor necesidad de los niños a justificar sus acciones mediante el uso de uno o varios de los mecanismos selectivos de desvinculación moral.

El punto crucial de estas diferencias en el uso de la desconexión moral reside justamente en la prevalencia de aspectos psicológicos y procesos de socialización en función del sexo, pues se ha encontrado con suficiente evidencia empírica (Caprara et al., 2008; Gómez Tabares, 2019b; Gómez-Tabares \& Narváez Marín, 2020; Valois et al., 2017) que la baja autoeficacia emocional y social, el déficit en habilidades sociales e interpersonales, la inestabilidad emocional, la poca empatía e insensibilidad emocional están asociados con los procesos de desactivación de los mecanismos sociocognitivos de regulación moral y son predictores de comportamientos disruptivos en la adolescencia. La relación entre conductas disruptivas y 
desconexión moral en adolescentes ha sido bastante estudiada (Bussey et al., 2015; D'Urso et al., 2018; Gini et al., 2014; Gini et al., 2015).

En este estudio se pudo corroborar parcialmente la segunda hipótesis (H2), al encontrar que los mecanismos de desconexión moral se correlacionaron directamente con el autodesprecio (autoestima negativa) y de manera inversa con la autoconfianza (autoestima positiva), lo que sugiere que la valía personal y la autoestima son factores que influyen en el uso de la desconexión moral. Sin embargo, estas dimensiones de valoración positiva y negativa del sí mismo no presentaron un efecto predictor en la desconexión moral.

La autoestima puede concebirse como un constructo psicológico global asociado a las respuestas emocionales de una persona respecto a sí mismo y al mundo social (Jordan et al., 2015). La autoestima es una característica personal relativamente duradera en gran parte del curso de la vida (Orth, 2018) y se refiere a los sentimientos y valoraciones positivas y negativas del yo (Li et al., 2018). También se han hecho distinciones entre la autoestima como constructo global unidimensional y las autovaloraciones específicas, las cuales implican una valoración cognitiva del desempeño de algún dominio, como lo son el autodesprecio o la autoconfianza (Jordan et al., 2015; Rosenberg, 1986). Dichas valoraciones influyen en la autoestima global en términos del concepto de sí mismo y el comportamiento social e interpersonal (Jordan et al., 2015).

Bajo estas distinciones conceptuales, el análisis de correlaciones sugiere que una autovaloración positiva de sí mismo o autoconfianza es un factor de protección de la desconexión moral, mientras que una autovaloración negativa o autodesprecio es un factor de riesgo. Al respecto, el estudio de Jordan et al. (2015) evidenció una asociación inversa entre las medidas de autoestima global y desconexión moral, mientras que en el estudio de Sticca et al. (2013) no se encontró dicha asociación. Asimismo, estudios similares sugieren que la desconexión moral y el uso de los mecanismos sociocognitivos particulares proporcionan una ruta de racionalización del comportamiento poco ético, lo que permite evitar la autocensura y mantener una valoración positiva de sí mismo y servir de amortiguador contra la disminución de la autoestima (Liang et al., 2020; Zhao et al., 2019).

Por otro lado, Jordan et al. (2015) consideran que el involucramiento en comportamientos poco éticos es un factor de estrés que afecta la valoración de sí mismo, debido a la disonancia cognitiva y al costo psicológico de la autoimagen moral. Estudios adicionales han presentado evidencia de que la autoestima es un predictor de la conducta social positiva y el razonamiento moral prosocial en los adolescentes (Laible et al., 2004; Padilla-Walker \& Carlo, 2014). Sin embargo, dada la escasez de estudios, la inconsistencia en los hallazgos disponibles y la falta de evidencia en esta investigación que indique efectos predictivos de las autovaloraciones positivas y negativas en la desconexión moral, es necesario realizar investigaciones adicionales para corroborar los datos encontrados y la hipótesis planteada.

Por otro lado, la tercera (H3) y cuarta (H4) hipótesis se lograron comprobar. Al respecto, se encontró que los mecanismos de desconexión moral se correlacionaron de manera directa con el autodesprecio (autoestima negativa), la impulsividad, la desesperanza, la ansiedad y, con menor fuerza, la depresión, lo que indica que son factores psicológicos de riesgo conducentes al uso de mecanismos sociocognitivos de desconexión moral en adolescentes. Dentro del conjunto de estas variables independientes, la impulsividad y el sexo presentaron los efectos predictores más altos de los mecanismos de desconexión moral. Así también, en el análisis de regresión, segmentado por hombres y mujeres, la impulsividad conservó su efecto predictor en cada uno de los mecanismos de desconexión moral. Estos hallazgos son coherentes con los estudios de Georgiou et al. (2020) y Gini et al. (2015), al encontrar evidencia empírica de que la conducta impulsiva-irresponsable y el sexo se asocian con la desconexión moral.

Tradicionalmente, la impulsividad en los adolescentes se ha asociado a la toma irreflexiva de decisiones y a actuar sin considerar las consecuencias. También involucra el procesamiento rápido de la información, la búsqueda de novedad y la habilidad restringida para aplazar la gratificación (Georgiou et al., 2020). Este amplio constructo también se encuentra asociado con pobres habilidades de planificación, dificultad para mantener la atención y el involucramiento en conductas de riesgo (Armstrong et al., 2020), entre ellas, el consumo de sustancias psicoactivas, el suicidio, la violencia física y otros tipos de comportamientos disruptivos. Este rasgo psicológico, caracterizado por el déficit en el control de impulsos, se asoció con el uso de los diferentes mecanismos de desconexión moral, tanto en hombres como mujeres, lo que sugiere que los adolescentes que tienden a actuar y pensar de manera impulsiva hacen un mayor uso de la desconexión moral y sus respectivos mecanismos para justificar sus acciones irreflexivas. Esta asociación entre los rasgos de impulsividad y la desconexión moral en adolescentes constituye un factor psicológico de 
vulnerabilidad para comportamientos agresivos y antisociales. En coherencia con los hallazgos, el déficit en la regulación de impulsos, las acciones temerarias e irreflexivas promueven el uso de la desconexión moral y sus diferentes mecanismos sociocognitivos de regulación para evitar la autocensura por dichas acciones, lo que es conducente a la agresión y la violencia, tal como se ha reportado en diversos estudios ya citados, que han mostrado una estrecha relación entre agresión, bullying, conductas antisociales, actividades delictivas y la desconexión moral en adolescentes. Si bien no hay elementos que sugieran que los adolescentes de este estudio presentan conductas violentas, sí es posible advertir que la impulsividad está asociada a mecanismos cognitivos que pueden conducir a justificar la agresión en el futuro y, por tanto, se estaría hablando de factores psicológicos de riesgo para la agresión.

Frente a la cuarta hipótesis (H4), se encontró que las variables clínicas internalizantes de ansiedad, desesperanza y depresión tuvieron un efecto predictor de la desconexión moral únicamente en las mujeres. A pesar de que en la literatura no se cuenta con estudios que permitan contrastar estas asociaciones en particular, sí se encuentran trabajos que muestran que los adolescentes, hombre y mujeres, presentan divergencias en cuanto a la prevalencia de rasgos comportamentales y psicosociales. Estudios psicológicos sugieren que las mujeres son más internalizantes, lo que significa que tienden más a la somatización, la ansiedad y la depresión, mientras que los hombres son mucho más externalizantes, lo que explicaría la tendencia a la agresión y la impulsividad (Côté et al., 2002; Gómez Tabares, 2019a; Plazas et al., 2010; Redondo Pacheco et al., 2015).

También, algunos autores (Dugatkin, 2006/2007; Zahn-Waxler et al., 1992) aluden a factores disposiciones a nivel biológico-evolutivo para explicar las diferencias en la expresión psicológica en función del sexo, mientras que posturas orientadas al aprendizaje social (Bandura, 1977; Gómez Tabares, 2019a) sugieren que estas expresiones psicológicas están asociadas a procesos vicarios y de modelamiento social que se establecen desde la crianza hasta los procesos de socialización que se dan en la escuela y en grupo de pares, con una fuerte influencia de la cultura a la cual se pertenece.

En cuanto a las asociaciones particulares de las variables clínicas en las mujeres, la desesperanza presentó un efecto en la predicción de los mecanismos de justificación moral, comparación ventajosa, deshumanización y distorsión de las consecuencias y la depresión, en los mecanismos de desplazamiento de la responsabilidad y la distorsión de las consecuencias. Los hallazgos particulares en las adolescentes mujeres parecen indicar que los indicadores de ansiedad y desesperanza están más involucrados en la justificación moral de la conducta o acciones dañinas contra sí mismas o los demás. En este sentido, a mayor ansiedad y desesperanza, mayor es la tendencia a usar los mecanismos del dominio de la conducta reprensible, con el fin de minimizar las conductas que se desprenden de los síntomas clínicos y, así, atenuar el malestar emocional. Por el contrario, la depresión está más asociada al uso de mecanismos de desplazamiento de la responsabilidad y distorsión de las consecuencias. Para Bandura (2002), el uso de estos mecanismos implica cuestionar y cambiar la percepción valorativa del efecto de la acción, con el fin de dirigir la responsabilidad a otra persona o grupo de ellas. Desde un punto de vista cognitivo, es usual encontrar en personas con síntomas de depresión filosofías orientadas a la devaluación del sí mismo, perdida de autoeficacia y valía personal, ideas distorsionadas, dogmáticas y absolutistas sobre el curso de la vida, el sí mismo y los demás (Beck, 1976; Ellis, 1973; Lega et al., 2013).

Una hipótesis para interpretar los hallazgos es que las adolescentes que presentan indicadores sintomáticos de depresión, ansiedad o desesperanza o presentan una tendencia a la impulsividad y una valoración negativa de sí mismas (autodesprecio), experimentan malestar emocional y, en consecuencia, recurren a la desconexión moral y sus diferentes mecanismos como una forma de justificar los síntomas o las acciones contra sí mismas o los demás. Bajo esta hipótesis se quiere plantear que, además de las creencias desadaptativas asociadas a cuadros clínicos de ansiedad, desesperanza y depresión, las acciones que se generen a raíz del malestar psicológico, por ejemplo, agresión auto y hetero dirigida, conductas de evitación, huida, automedicación y consumo de sustancias psicoactivas, usuales en la ansiedad, o conductas de aislamiento social, autolesiones no suicidas, agresión y comportamientos suicidas, asociados a cuadros de desesperanza y depresión, involucrarían el uso de mecanismos de desconexión moral, con el fin de evitar la autocensura por sus propias acciones. Se trata, entonces, de mecanismos cognitivos que limitarían la capacidad de agencia y autoeficacia de las adolescentes sobre su propio malestar psicológico y, por tanto, promoverían acciones que van en contra del bienestar de sí mismas y otras personas.

Esta hipótesis nace de un antecedente teórico en la terapia racional emotiva conductual, respecto de la formación de creencias de segundo orden o síntomas perturbadores secundarios (Lega et al., 2013). Consiste 
en que la presentación de ansiedad o depresión clínicamente significativa, resultado de ideas irracionales, genera nuevos acontecimientos activantes, por ejemplo, sentir depresión por estar deprimido, o sentir ansiedad por ser ansioso. Bajo esta idea, se argumenta que, para evitar la formación de síntomas secundarios, por ejemplo, la autocensura y la culpa por sentir ansiedad o desesperanza o por las acciones en contra de sí o los demás, se recurre a la desconexión moral. Dicho de otro modo, la persona interpreta las razones posibles de las experiencias de depresión, desesperanza o ansiedad y bajo esta lectura de los síntomas podría recurrir a la desconexión moral para así evitar los síntomas secundarios que generan malestar emocional.

Evidentemente, las hipótesis planteadas en cuanto a la interpretación de los hallazgos de este estudio sugieren mayor búsqueda de evidencias, por lo que se requiere de un mayor desarrollo teórico y nuevas exploraciones investigativas en esta línea de trabajo. En la medida en que se cuente con mayor soporte empírico, se podrán confirmar o rechazar estos hallazgos y las hipótesis planteadas.

Se deben señalar algunas limitaciones del presente estudio. Primero, al usar un diseño transversal y contar con una muestra no clínica y relativamente pequeña, los resultados no pueden ser generalizados a una comunidad en general o a muestras con diagnóstico clínico. Segundo, la identificación de los factores clínico-psicológicos asociados a la desconexión moral y cada mecanismo particular tiene un sustento desde los correlatos estadísticos, pero carecen de evidencia longitudinal. Finalmente, los datos de este estudio fueron obtenidos mediante medidas de autoinforme, por lo que no están exentos de sesgos de conveniencia o deseabilidad social por parte de los participantes.

\section{Referencias}

Alarcón Parco, D. \& Bárrig Jó, P. S. (2015). Conductas internalizantes y externalizantes en adolescentes. Liberabit, 21(2), 253-259. http://ojs3.revistaliberabit.com/index.php/Liberabit/article/view/269/144

Armstrong, T. A., Boisvert, D., Wells, J. \& Lewis, R. (2020). Extending Steinberg's adolescent model of risk taking to the explanation of crime and delinquency: Are impulsivity and sensation seeking enough? Personality and Individual Differences, 165, Article 110133. https://doi.org/10.1016/j.paid.2020.110133

Bandura, A. (1977). Social learning theory (Vol. 1). Prentice Hall. https://doi.org/10.1177/105960117700200317

Bandura, A. (1990). Selective activation and disengagement of moral control. Journal of Social Issues, 46(1), 27-46. https://doi.org/10.1111/j.1540-4560.1990.tb00270.x

Bandura, A. (1991). Social cognitive theory of moral thought and action. En W. M. Kurtines \& J. L. Gewirtz (Eds.), Handbook of moral behavior and development, Volume 1: Theory (pp. 45-103). Lawrence Erlbaum.

Bandura, A. (1999). Moral disengagement in the perpetration of inhumanities. Personality and Social Psychology Review, 3(3), 193209. https://doi.org/10.1207/s15327957pspr0303_3

Bandura, A. (2002). Selective moral disengagement in the exercise of moral agency. Journal of Moral Education, 31(2), 101-119. https://doi.org/10.1080/0305724022014322

Bandura, A. (2016). Moral disengagement: How people do harm and live with themselves. Worth Publishers.

Bandura, A., Barbaranelli, C., Caprara, G. V. \& Pastorelli, C. (1996). Mechanisms of moral disengagement in the exercise of moral agency. Journal of Personality and Social Psychology, 71(2), 364-374. https://doi.org/10.1037/0022-3514.71.2.364

Bandura, A., Underwood, B. \& Fromson, M. E. (1975). Disinhibition of aggression through diffusion of responsibility and dehumanization of victims. Journal of Research in Personality, 9(4), 253-269. https://doi.org/10.1016/0092-6566(75)90001-X

Beck, A. T. (1976). Cognitive therapy and the emotional disorders. International Universities Press.

Beck, A. T., Epstein, N., Brown, G. \& Steer, R. A. (1988). An inventory for measuring clinical anxiety: Psychometric properties. Journal of Consulting and Clinical Psychology, 56(6), 893-897. https://doi.org/10.1037/0022-006X.56.6.893

Beck, A. T., Rush, A. J., Shaw, B. F. \& Emery, G. (1979). Cognitive therapy of depression. Guilford Press.

Beck, A. T., Weissman, A., Lester, D. \& Trexler, L. (1974). The measurement of pessimism: The Hopelessness Scale. Journal of Consulting and Clinical Psychology, 42(6), 861-865. https://doi.org/10.1037/h0037562

Bjärehed, M., Thornberg, R., Wänström, L. \& Gini, G. (2019). Individual moral disengagement and bullying among Swedish fifth graders: The role of collective moral disengagement and pro-bullying behavior within classrooms. Journal of Interpersonal Violence, 36(17-18), NP9576-NP9600. https://doi.org/10.1177/0886260519860889

Bussey, K., Quinn, C. \& Dobson, J. (2015). The moderating role of empathic concern and perspective taking on the relationship between moral disengagement and aggression. Merrill-Palmer Quarterly, 61(1), 10-29. https://doi.org/10.13110/merrpalmquar1982.61.1.0010

Campos, J., Barbosa-Ducharne, M., Dias, P., Rodrigues, S., Martins, A. C. \& Leal, M. (2019). Emotional and behavioral problems and psychosocial skills in adolescents in residential care. Child and Adolescent Social Work Journal, 36(3), 237246. https://doi.org/10.1007/s10560-018-0594-9

Caprara, G. V., Di Giunta, L., Eisenberg, N., Gerbino, M., Pastorelli, C. \& Tramontano, C. (2008). Assessing regulatory emotional selfefficacy in three countries. Psychological Assessment, 20(3), 227-237. https://doi.org/10.1037/1040-3590.20.3.227

Ceballos-Ospino, G. A., Suarez-Colorado, Y., Suescún-Arregocés, J., Gamarra-Vega, L. M., González, K. E. \& Sotelo-Manjarres, A. P. (2015). Ideación suicida, depresión y autoestima en adolescentes escolares de Santa Marta. Duazary: Revista Internacional de Ciencias de la Salud, 12(1), 15-22. https://doi.org/10.21676/2389783X.1394

Chahín Pinzón, N., Moncada Duarte, C. E. \& Acosta Salazar, H. U. (2019). Estudio de las propiedades psicométricas de la Escala Barratt de Impulsividad (BIS-11) en niños y adolescentes. Terapia Psicológica, 37(2), 129-140. https://doi.org/10.4067/S0718$\underline{48082019000200129}$ 
Cogollo, Z., Campo-Arias, A. \& Herazo, E. (2015). Escala de Rosenberg para autoestima: consistencia interna y dimensionalidad en estudiantes de Cartagena, Colombia. Psychologia: Avances de la Disciplina, 9(2), 61-71. https://doi.org/10.21500/19002386.1814

Cohen, J. (1988). Statistical power analysis for the behavioral sciences (2a ed.). Lawrence Erlbaum.

Correa Duque, M. C. (2017). Aproximaciones epistemológicas y conceptuales de la conducta prosocial. Zona Próxima, 27 , Artículo 3. https://doi.org/10.14482/zp.27.10978

Côté, S., Tremblay, R. E., Nagin, D., Zoccolillo, M. \& Vitaro, F. (2002). The development of impulsivity, fearfulness, and helpfulness during childhood: Patterns of consistency and change in the trajectories of boys and girls. The Journal of Child Psychology and Psychiatry, 43(5), 609-618. https://doi.org/10.1111/1469-7610.00050

De Caroli, M. E., Sagone, E. \& Falanga, R. (2011). Civic moral disengagement and personality. A comparison between law and psychology Italian students. International Journal of Developmental and Educational Psychology, 5(1), 105-112. https://www.redalyc.org/pdf/3498/349832343010.pdf

Doyle, F. L. \& Bussey, K. (2018). Moral disengagement and children's propensity to tell coached lies. Journal of Moral Education, 47(1), 91-103. https://doi.org/10.1080/03057240.2017.1380611

Dugatkin, L. A. (2007). Qué es el altruismo: la búsqueda científica del origen de la generosidad (E. Marengo, Trad.). Katz Editores. (Obra original publicada en 2006).

D'Urso, G., Petruccelli, I. \& Pace, U. (2018). Drug use as a risk factor of moral disengagement: A study on drug traffickers and offenders against other persons. Psychiatry, Psychology and Law, 25(3), 417-424. https://doi.org/10.1080/13218719.2018.1437092

Ellis, A. (1973). Humanistic psychotherapy: The rational-emotive approach. Julian Press.

Frisby-Osman, S. \& Wood, J. L. (2020). Rethinking how we view gang members: An examination into affective, behavioral, and mental health predictors of UK gang-involved youth. Youth Justice, 20(1-2), 93-112. https://doi.org/10.1177/1473225419893779

Georgiou, S. N., Charalambous, K. \& Stavrinides, P. (2020). Mindfulness, impulsivity, and moral disengagement as parameters of bullying and victimization at school. Aggressive Behavior, 46(1), 107-115. https://doi.org/10.1002/ab.21876

Gini, G. (2006). Social cognition and moral cognition in bullying: What's wrong? Aggressive Behavior, 32(6), 528-539. https://doi.org/10.1002/ab.20153

Gini, G., Pozzoli, T. \& Bussey, K. (2015). Moral disengagement moderates the link between psychopathic traits and aggressive behavior among early adolescents. Merrill-Palmer Quarterly, 61(1), 51-67. https://doi.org/10.13110/merrpalmquar1982.61.1.0051

Gini, G., Pozzoli, T. \& Hauser, M. (2011). Bullies have enhanced moral competence to judge relative to victims, but lack moral compassion. Personality and Individual Differences, 50(5), 603-608. https://doi.org/10.1016/j.paid.2010.12.002

Gini, G., Pozzoli, T. \& Hymel, S. (2014). Moral disengagement among children and youth: A meta-analytic review of links to aggressive behavior. Aggressive Behavior, 40(1), 56-68. https://doi.org/10.1002/ab.21502

Gómez Etayo, E., Núñez, C. \& Gallo Consuegra, L. (2020). Género y sexualidad juvenil. Amor, relacionamientos y concepciones sobre los géneros. En C. Núñez \& E. Gómez Etayo (Eds.), Juventud, violencia y paz. Experiencias investigativas en barriadas populares de Cali y Medellín (pp. 211-231). Sello Editorial Universidad de Medellín. https://repository.udem.edu.co/handle/11407/6196

Gómez Etayo, E., Núñez, C., Giraldo-Rojas, J., Gómez Tabares, A. S. \& Arango Vásquez, A. (2020). Jóvenes, violencia y paz. Contexto e intencionalidad. En C. Núñez \& E. Gómez Etayo (Eds.), Juventud, violencia y paz. Experiencias investigativas en barriadas populares de Cali y Medellín (pp. 19-47). Sello Editorial Universidad de Medellín. https://repository.udem.edu.co/handle/11407/6190

Gómez-Lugo, M., Espada, J. P., Morales, A., Marchal-Bertrand, L., Soler, F. \& Vallejo-Medina, P. (2016). Adaptation, validation, reliability and factorial equivalence of the Rosenberg Self-Esteem Scale in Colombian and Spanish population. The Spanish Journal of Psychology, 19, Artículo E66. https://doi.org/10.1017/sjp.2016.67

Gómez Tabares, A. S. (2019a). Prosocialidad. Estado actual de la investigación en Colombia. Revista Colombiana de Ciencias Sociales, 10(1), 188-218. https://doi.org/10.21501/22161201.3065

Gómez Tabares, A. S. (2019b). Conductas prosociales y su relación con la empatía y la autoeficacia para la regulación emocional en adolescentes desvinculados de grupos armados ilegales. Revista Criminalidad, 61(3), 221-246. https://www.policia.gov.co/file/223753/download?token=JEAfxABX

Gómez Tabares, A. S. \& Durán Palacio, N. M. (2020). Motivaciones prosociales, empatía y diferencias de género en adolescentes víctimas del conflicto armado e infractores de la ley. Revista sobre la Infancia y la Adolescencia, 18, 69-90. https://doi.org/10.4995/reinad.2020.12771

Gómez Tabares, A. S. \& Landinez-Martinez, D. (2021). Moral disengagement mechanisms and its relationship with aggression and bullying behaviour among school children and youth at psychosocial risk. Emotional and Behavioural Difficulties, 1-15. https://doi.org/10.1080/13632752.2021.1945801

Gómez Tabares, A. S. \& Narváez Marín, M. (2019). Mecanismos de desconexión moral y su relación con la empatía y la prosocialidad en adolescentes que han tenido experiencias delictivas. Revista de Psicología de la Pontificia Universidad Católica del Perú, 37(2), 603-641. https://doi.org/10.18800/psico.201902.010

Gómez-Tabares, A. S. \& Narváez Marín, M. (2020). Tendencias prosociales y su relación con la empatía y la autoeficacia emocional en adolescentes en vulnerabilidad psicosocial. Revista Colombiana de Psicología, 29(2), 125-147. https://doi.org/10.15446/rcp.v29n2.78430

Gómez Tabares, A. S., Narváez Marín, M. \& Correa Duque, M. C. (2019). Motivaciones prosociales y desconexión moral en adolescentes desvinculados de grupos armados ilegales. Psicología desde el Caribe, 36(3), 297-327. https://doi.org/10.14482/psdc.36.3.303.6

Gómez Tabares, A. S., Núñez, C., Agudelo Osorio, M. P. \& Grisales Aguirre, A. M. (2020). Riesgo e ideación suicida y su relación con la impulsividad y la depresión en adolescentes escolares. Revista Iberoamericana de Diagnóstico y Evaluación/e Avaliação Psicológica, 54, 147-163. https://doi.org/10.21865/RIDEP54.1.12

Gómez Tabares, A., Núñez, C., Arango Vásquez, A. \& Giraldo-Rojas, J. (2020). La prosocialidad como categoría de discusión en escenarios de reconciliación y construcción de paz. En C. Núñez \& E. Gómez Etayo (Eds.), Juventud, violencia y paz. Experiencias investigativas en barriadas populares de Cali y Medellín (pp. 125-154). Sello Editorial Universidad de Medellín. https://repository.udem.edu.co/handle/11407/6193

Gómez Tabares, A. S., Núñez, C., Caballo, V. E., Agudelo Osorio, M. P. \& Grisales Aguirre, A. M. (2019). Predictores psicológicos del riesgo suicida en estudiantes universitarios. Psicología Conductual, 27(3), 391-413. https://www.behavioralpsycho.com/wpcontent/uploads/2019/12/03.Gomez-27-3oa-1.pdf

González Cifuentes, C. E. (2009). Propiedades psicométricas de la Escala de Desesperanza de Beck en una muestra bogotana. Psychologia, 3(2), 17-30. https://biblat.unam.mx/hevila/PsychologiaAvancesdeladisciplina/2009/vol3/no2/1.pdf 
Gutzwiller-Helfenfinger, E. (2015). Moral disengagement and aggression: Comments on the special issue. Merrill-Palmer Quarterly, 61(1), 192-211. https://doi.org/10.13110/merrpalmquar1982.61.1.0192

Hardy, S. A., Bean, D. S. \& Olsen, J. A. (2015). Moral identity and adolescent prosocial and antisocial behaviors: Interactions with moral disengagement and self-regulation. Journal of Youth and Adolescence, 44(8), 1542-1554. https://doi.org/10.1007/s10964-014-0172-1

Hernández Sampieri, R., Fernández Collado, C. \& Baptista Lucio, P. (2014). Metodología de la investigación (6ª ed). McGraw Hill.

Hyde, L. W., Shaw, D. S. \& Moilanen, K. L. (2010). Developmental precursors of moral disengagement and the role of moral disengagement in the development of antisocial behavior. Journal of Abnormal Child Psychology, 38(2), 197-209. https://doi.org/10.1007/s10802-009-9358-5

Hymel, S. \& Perren, S. (2015). Introduction to the special issue: Moral disengagement and aggression in children and youth. MerrillPalmer Quarterly, 61(1), 1-9. https://doi.org/10.13110/merrpalmquar1982.61.1.0001

Jordan, J., Leliveld, M. C. \& Tenbrunsel, A. E. (2015). The Moral Self-Image Scale: Measuring and understanding the malleability of the moral self. Frontiers in Psychology, 6, Artículo 1878. https://doi.org/10.3389/fpsyg.2015.01878

Kokkinos, C. M. \& Kipritsi, E. (2018). Bullying, moral disengagement and empathy: Exploring the links among early adolescents. Educational Psychology, 38(4), 535-552. https://doi.org/10.1080/01443410.2017.1363376

Kollerová, L., Soukup, P. \& Gini, G. (2018). Classroom Collective Moral Disengagement Scale: Validation in Czech adolescents. European Journal of Developmental Psychology, 15(2), 184-191. https://doi.org/10.1080/17405629.2017.1292907

Laible, D. J., Carlo, G. \& Roesch, S. C. (2004). Pathways to self-esteem in late adolescence: The role of parent and peer attachment, empathy, and social behaviours. Journal of Adolescence, 27, 703-716. https://doi.org/10.1016/j.adolescence.2004.05.005

Lega, L., Caballo, V. \& Ellis, A. (2013). Teoría y práctica de la terapia racional emotivo-conductual. Siglo XXI.

Li, J., Hao, J. \& Shi, B. (2018). From moral judgments to prosocial behavior: Multiple pathways in adolescents and different pathways in boys and girls. Personality and Individual Differences, 134, 149-154. https://doi.org/10.1016/j.paid.2018.06.017

Liang, Y., Liu, L., Tan, X., Dang, J., Li, C. \& Gu, Z. (2020). The moderating effect of general system justification on the relationship between unethical behavior and self-esteem. Self and Identity, 19(2), 140-163. https://doi.org/10.1080/15298868.2018.1541328

Magán, I., Sanz, J. \& García-Vera, M. P. (2008). Psychometric properties of a Spanish version of Beck Anxiety Inventory (BAI) in general population. The Spanish Journal of Psychology, 11(2), 626-640. https://doi.org/10.1017/S1138741600004637

Mestre, M. V., Samper, P., Frías, M. D. \& Tur, A. M. (2009). Are women more empathetic than men? A longitudinal study in adolescence. The Spanish Journal of Psychology, 12(1), 76-83. https://doi.org/10.1017/S1138741600001499

Núñez, C., Arango Vásquez, A., Giraldo-Rojas, J., Gómez Tabares, A. S. \& Gómez Etayo, E. (2020). Justicia restaurativa, cultura de paz y competencias psicosociales. En C. Núñez \& E. Gómez Etayo (Eds.), Juventud, violencia y paz. Experiencias investigativas en barriadas populares de Cali y Medellín (pp. 77-123). Sello Editorial Universidad de Medellín. https://repository.udem.edu.co/handle/11407/6192

Núñez, C., Gallo Consuegra, L., Gómez Etayo, E., Carantón Sánchez, J., Vargas Zuluaga, N. M. \& Gómez Tabares, A. S. (2020). Vivir el contexto: exigencias y relaciones contrapuestas en jóvenes. En C. Núñez \& E. Gómez Etayo (Eds.), Juventud, violencia y paz. Experiencias investigativas en barriadas populares de Cali y Medellín (pp. 157-188). Sello Editorial Universidad de Medellín. https://repository.udem.edu.co/handle/11407/6194

Núñez, C., Gallo Consuegra, L., Gómez Etayo, E., Vargas Zuluaga, N. M., Carantón Sánchez, J. \& Gómez Tabares, A. S. (2020). Conceptos de experiencia humana, jóvenes y violencia. En C. Núñez \& E. Gómez Etayo (Eds.), Juventud, violencia y paz. Experiencias investigativas en barriadas populares de Cali y Medellín (pp. 49-76). Sello Editorial Universidad de Medellín. https://repository.udem.edu.co/handle/11407/6191

Núñez Rojas, A. C., Tobón Tobón, S., Arias Henao, D. \& Bañol Ramírez, J. E. (2006). Sistema articulado de bienestar universitario. Hacia la Promoción de la Salud, 11, 61-72. http://www.redalyc.org/articulo.oa?id=309126325008

Oquendo, M. A., Baca García, E., Graver, R., Morales, M., Montalvan, V. \& Mann, J. (2001). Spanish adaption of the Barratt Impulsiveness Scale (BIS-11). The European Journal of Psychiatry, 15(3), 147-155. http://pascalfrancis.inist.fr/vibad/index.php?action=getRecordDetail\&idt=13780488

Oquendo, M. A., Galfalvy, H., Russo, S., Ellis, S. P., Grunebaum, M. F., Burke, A. \& Mann, J. J. (2004). Prospective study of clinical predictors of suicidal acts after a major depressive episode in patients with major depressive disorder or bipolar disorder. American Journal of Psychiatry, 161(8), 1433-1441. https://doi.org/10.1176/appi.ajp.161.8.1433

Orth, U. (2018). The family environment in early childhood has a long-term effect on self-esteem: A longitudinal study from birth to age 27 years. Journal of Personality and Social Psychology, 114(4), 637-655. https://doi.org/10.1037/pspp0000143

Padilla-Walker, L. M. \& Carlo, G. (2014). The study of prosocial behavior: Past, present, and future. En L. M. Padilla-Walker \& G. Carlo (Eds.), Prosocial development: A multidimensional approach (pp. 3-16). Oxford University Press. https://doi.org/10.1093/acprof:oso/9780199964772.003.0001

Patton, J. H., Stanford, M. S. \& Barratt, E. S. (1995). Factor structure of the Barratt Impulsiveness Scale. Journal of Clinical Psychology, 51(6), 768-774. https://doi.org/10.1002/1097-4679(199511)51:6\%3C768::aid-jclp2270510607\%3E3.0.co;2-1

Petruccelli, I., Simonelli, C., Barbaranelli, C., Grilli, S., Tripodi, M. F. \& D'Urso, G. (2017). Moral disengagement strategies in sex offenders. Psychiatry, Psychology and Law, 24(3), 470-480. https://doi.org/10.1080/13218719.2016.1252291

Plazas, E. A., Morón Cotes, M. L., Santiago, A., Sarmiento, H., Ariza López, S. E. \& Patiño, C. D. (2010). Relaciones entre iguales, conducta prosocial y género desde la educación primaria hasta la universitaria en Colombia. Universitas Psychologica, 9(2), 357369. https://doi.org/10.11144/Javeriana.upsy9-2.ricp

Quinn, C. A. \& Bussey, K. (2015a). Moral disengagement, anticipated social outcomes and adolescents' alcohol use: Parallel latent growth curve analyses. Journal of Youth and Adolescence, 44(10), 1854-1870. https://doi.org/10.1007/s10964-015-0345-6

Quinn, C. A. \& Bussey, K. (2015b). The role of moral disengagement in underage drinking and heavy episodic drinking. Substance Use \& Misuse, 50(11), 1437-1448. https://doi.org/10.3109/10826084.2015.1018541

Raskauskas, J. L., Gregory, J., Harvey, S. T., Rifshana, F. \& Evans, I. M. (2010). Bullying among primary school children in New Zealand: Relationships with prosocial behaviour and classroom climate. Educational Research, 52, $1-13$. https://doi.org/10.1080/00131881003588097

Redondo Pacheco, J., Rangel Noriega, K. J. \& Luzardo Briceño, M. (2015). Diferencias en comportamientos prosociales entre adolescentes colombianos. Psicogente, 18(34), 311-319. https://doi.org/10.17081/psico.18.34.507

Rescorla, L. A., Blumenfeld, M. C., Ivanova, M. Y., Achenbach, T. M. \& International ASEBA Consortium. (2019). International comparisons of the dysregulation profile based on reports by parents, adolescents, and teachers. Journal of Clinical Child \& Adolescent Psychology, 48(6), 866-880. https://doi.org/10.1080/15374416.2018.1469090 
Rosenberg, M. (1965). Society and the adolescent self-image. Princeton University Press.

Rosenberg, M. (1986). Self-concept from middle childhood through adolescence. En J. Suls \& A. G. Greenwald (Eds.), Psychological perspectives on the self. Volume 2 (pp. 107-135). Lawrence Erlbaum.

Rubio-Garay, F., Amor, P. J. \& Carrasco, M. A. (2017). Dimensionality and psychometric properties of the Spanish version of the Mechanisms of Moral Disengagement Scale (MMDS-S). Revista de Psicopatología y Psicología Clínica, 22(1), 43-54. https://doi.org/10.5944/rppc.vol.22.num.1.2017.16014

Rueda-Jaimes, G. E., Castro-Rueda, V. A., Rangel-Martínez-Villalba, A. M., Moreno-Quijano, C., Martínez-Salazar, G. A. \& Camacho, P. A. (2018). Validación de la Escala de Desesperanza de Beck en pacientes con riesgo suicida. Revista de Psiquiatría y Salud Mental, 11(2), 86-93. https://doi.org/10.1016/j.rpsm.2016.09.004

Sanz, J., Gutiérrez, S., Gesteira, C. \& García-Vera, M. P. (2014). Criterios y baremos para interpretar el "Inventario de Depresión de BeckII" (BDI-II). Psicología Conductual, 22(1), 37-59. https://www.behavioralpsycho.com/wp-content/uploads/2019/08/03.Sanz 22-1oa.pdf

Sanz, J. \& Vázquez, C. (1998). Fiabilidad, validez y datos normativos del Inventario para la Depresión de Beck. Psicothema, 10(2), 303-318. http://psicothema.com/pdf/167.pdf

Se establecen las normas científicas, técnicas y administrativas para la investigación en salud. Resolución 8430, Ministerio de Salud de Colombia (1993). https://www.minsalud.gov.co/sites/rid/Lists/BibliotecaDigital/RIDE/DE/DIJ/RESOLUCION-8430-DE-1993.PDF

Se reglamenta el ejercicio de la profesión de psicología, se dicta el Código Deontológico y Bioético y otras disposiciones, Ley 1090, Congreso de Colombia (2006). https://www.funcionpublica.gov.co/eva/gestornormativo/norma_pdf.php?i=66205

Sticca, F., Ruggieri, S., Alsaker, F. \& Perren, S. (2013). Longitudinal risk factors for cyberbullying in adolescence. Journal of Community \& Applied Social Psychology, 23(1), 52-67. https://doi.org/10.1002/casp.2136

Thornberg, R. \& Jungert, T. (2014). School bullying and the mechanisms of moral disengagement. Aggressive Behavior, 40(2), 99-108. https://doi.org/10.1002/ab.21509

Tillman, C. J., Gonzalez, K., Whitman, M. V., Crawford, W. S. \& Hood, A. C. (2017). A multi-functional view of moral disengagement: Exploring the effects of learning the consequences. Frontiers in Psychology, 8, Artículo 2286. https://doi.org/10.3389/fpsyg.2017.02286

Urrego Barbosa, S. C., Valencia Casallas, O. L. \& Villalba, J. (2017). Validación de la Escala Barrat de Impulsividad (BIS-11) en población bogotana. Diversitas, 13(2), 143-157. https://doi.org/10.15332/s1794-9998.2017.0002.01

Valois, R. F., Zullig, K. J. \& Revels, A. A. (2017). Aggressive and violent behavior and emotional self-efficacy: Is there a relationship for adolescents? Journal of School Health, 87(4), 269-277. https://doi.org/10.1111/josh.12493

Van der Graaff, J., Branje, S., De Wied, M., Hawk, S., Van Lier, P. \& Meeus, W. (2014). Perspective taking and empathic concern in adolescence: Gender differences in developmental changes. Developmental Psychology,50(3), 881888. https://doi.org/10.1037/a0034325

Van der Graaff, J., Carlo, G., Crocetti, E., Koot, H. M. \& Branje, S. (2018). Prosocial behavior in adolescence: Gender differences in development and links with empathy. Journal of Youth and Adolescence, 47(5), 1086-1099. https://doi.org/10.1007/s10964-017-0786-1

Van Noorden, T. H. J., Haselager, G. J. T., Cillessen, A. H. N. \& Bukowski, W. M. (2014). Dehumanization in children: The link with moral disengagement in bullying and victimization. Aggressive Behavior, 40(4), 320-328. https://doi.org/10.1002/ab.21522

Villegas de Posada, C., Flórez, J. \& Espinel, N. (2018). Moral disengagement mechanisms and armed violence. A comparative study of paramilitaries and guerrillas in Colombia. Revista Colombiana de Psicología, 27(1), 55-69. https://doi.org/10.15446/rcp.v27n1.6219

Zahn-Waxler, C., Radke-Yarrow, M., Wagner, E. \& Chapman, M. (1992). Development of concern for others. Developmental Psychology, 28(1), 126-136. https://doi.org/10.1037/0012-1649.28.1.126

Zhao, H., Zhang, H. \& Xu, Y. (2019). Effects of perceived descriptive norms on corrupt intention: The mediating role of moral disengagement. International Journal of Psychology, 54(1), 93-101. https://doi.org/10.1002/ijop.12401

Fecha de recepción: Enero de 2020.

Fecha de aceptación: Noviembre de 2020. 